\title{
The diagnostic and prognostic significance of small nuclear ribonucleoprotein Sm D1 aberrantly high expression in hepatocellular carcinoma
}

\author{
Huaxiang Wang ${ }^{1 *}$, Fengfeng $\mathrm{Xu}^{1,2^{*}}$, Lingling $\mathrm{Lu}^{1 *}$, Fang Yang ${ }^{1,2}$, Xinghua Huang ${ }^{1,2}$, Lizhi Lv ${ }^{1,2}$, Huanzhang \\ $\mathrm{Hu}^{1,2}{ }^{\bowtie}, \mathrm{Yi}_{\text {Jiang }}{ }^{1,2}$ \\ 1. The Fuzong Clinical Medical College of Fujian Medical University, Fuzhou, Fujian 350025, PR China. \\ 2. Department of Hepatobiliary Surgery, 900 Hospital of the Joint Logistic Team, Fuzhou, Fujian 350025, PR China. \\ * Contributed equally. \\ $\square$ Corresponding authors: Department of Hepatobiliary Surgery, 900 Hospital of the Joint Logistics Team, No. 156 The Second West Ring Road, Fuzhou, Fu Jian \\ Province 350025, PR China. E-mail addresses: jiangyi1963@126.com (Yi Jiang), hhzhang2000@163.com (Huanzhang Hu).
}

() The author(s). This is an open access article distributed under the terms of the Creative Commons Attribution License (https://creativecommons.org/licenses/by/4.0/). See http://ivyspring.com/terms for full terms and conditions.

Received: 2021.07.21; Accepted: 2021.11.09; Published: 2022.01.01

\begin{abstract}
Small nuclear ribonucleoprotein Sm DI (SNRPDI), one of the crucial genes encoding core spliceosome components, was abnormally highly expressed in multiple types of tumors. In this study, we investigated the diagnostic and prognostic significance of SNRPDI in hepatocellular carcinoma (HCC). The investigation of datasets from GEO and TCGA databases revealed that SNRPDI expression in HCC was significantly higher than adjacent normal liver tissues, which was validated by immunohistochemistry (IHC). Both GO, KEGG analysis showed that the SNRPDI co-expressed genes mainly enriched in Cell division, Nuclear import, mRNA splicing via spliceosome, Ribosome, Cell cycle, etc. Survival analysis from the GSE14520 dataset and 154 HCC cohorts exhibited a significant association of high SNRPDI expression with poor overall survival and recurrence-free survival. ROC analysis showed that the abnormally high SNRPDI mRNA expression has diagnostic significance in distinguishing between HCC and normal liver tissue (AUC = 0.819). Gene set enrichment analysis (GSEA) demonstrated that the high expression of SNRPDI might regulate HCC tumorigenesis and progression by affecting the cell cycle, mismatch repair, DNA replication, and RNA degradation, etc. The luciferase report assay revealed that SNRPDI was the direct target gene of miR-100 manifested by decreased SNRPDI expression and luciferase activity in the HCC cells upon miR-100 overexpression. Finally, SNRPDI may as an oncogene affecting the progression of HCC through regulates the mTOR pathway and autophagy.
\end{abstract}

Key words: SNRPD1, miR-100, Hepatocellular carcinoma, prognosis, mechanism.

\section{Introduction}

Hepatocellular carcinoma (HCC) causes a huge health-related economic burden globally, especially in East Asia and sub-Saharan Africa [1]. Chronic hepatitis virus infections such as hepatitis B virus (HBV) and hepatitis $\mathrm{C}$ virus (HCV), long-term exposure to aflatoxins and chemical substances, alcohol consumption, and non-alcoholic fatty liver disease are high-risk factors for HCC [1, 2]. Serum alpha-fetoprotein (AFP), the golden standard prognostic biomarker currently of HCC patients, has a low sensitivity and specificity due to was easily influenced by none-HCC disease. Therefore, in-depth investigation of the molecular mechanism and signaling pathways of the occurrence and development of HCC and the search for highly sensitive biomarkers are of great significance for the early diagnosis and prognosis of HCC.

Spliceosome, a dynamic macromolecular complex composed of five small nuclear ribonucleoproteins (termed U1, U2, U4, U5, and U6) and a set of proteins associated with spliceosome, was responsible for participating in the splicing of the 
pre-messenger RNA, such as removing introns and then the connection of exons in a certain order [3-5]. Each small nuclear ribonucleoprotein was composed of small nuclear RNA and a set of 7 SNRP proteins (B/B', D1, D2, D3, E, F, and G) [6]. These SNRP proteins form a specific structure to encompass the RNA and to share a conserved SNRP domain which undergoes responsible for the assembling of snRNA in the order sequence [7]. The process of splicing on the pre-mRNA plays a pivotal role in the expression of many genes $[8,9]$. Mutations in splicing factors or alterations in the expression of splicing mechanical elements will affect the splicing patterns, which may lead to the occurrence of many tumors [10-12]. Small Nuclear Ribonucleoprotein Polypeptides B and E (SNRPB, SNRPE), both the core components of the spliceosome, were significantly overexpressed in HCC tissues and associated with worse prognosis [13, 14]. Additionally, SNRPB has been reported as a prognostic marker for non-small cell lung cancer, cervical cancer, glioma, etc [15-17]. SNRPD1 was a crucial gene that regulates the assembly of the pluripotency-specific spliceosome and acquires and maintains pluripotency, and its high protein expression in somatic cells was related to kidney damage and pulmonary hypertension in patients with systemic lupus erythematosus [18, 19]. It has been reported that SNRPD1 was significantly overexpressed in neuroblastoma and was an important therapeutic target through regulating the process of mitosis [20]. However, no previous studies have investigated the role of SNRPD1 and its prognostic value in HCC.

In this present study, we analyze the mRNA and protein expression of SNRPD1 in the Gene Expression Omnibus (GEO) and the Human Protein Atlas databases, respectively. We explored the gene function by performing gene ontology (GO) and The Kyoto Encyclopedia of Genes and Genomes (KEGG) analysis for co-expressed genes of SNRPD1 [21]. Next, we investigated the association between the SNRPD1 expression and clinical outcomes in the GSE14520 dataset and 154 HCC cohorts. Then, the luciferase report assay revealed SNRPD1 is the direct target gene of miR-100. Finally, SNRPD1 may be an oncogene to impact the occurrence and development of HCC by regulating the mTOR signaling pathway and autophagy.

\section{Materials and methods}

\subsection{Gene expression analysis}

Gene Expression Profiling Interactive Analysis (GEPIA), a Web server for analysis of the mRNA expression data from The Cancer Genome Atlas
(TCGA), was used to analyze the expressed distribution of SNRPD1 in BodyMap and analyze the association of the SNRPD1 expression with tumor stages and survival [22]. We used the TNMplot database to comprise SNRPD1 expression in normal, tumor, and metastatic tissues of HCC patients. The Cancer Cell Line Encyclopedia project (CCLE) database was used to investigate the association of SNRPD1 mRNA levels with different liver cancer cell lines. We explore the SNRPD1 protein expression in HCC and adjacent normal tissues from the Human Protein Atlas database [23]. We queried the genetic alteration information of the SNRPD1 in cBioPortal for Cancer Genomics and analyzed the correlations between SNRPD1 alteration with prognosis [24].

\subsection{Prognostic analysis using SNRPDI expression and clinicopathological data in HCC patients.}

We downloaded the GSE14520 dataset from the GEO database to study the relationship between the expression of SNRPD1 and the clinical outcome of HCC patients [25]. Overall survival (OS) was defined as the time interval between surgery and death or between surgery and the last observation point. Recurrence-free survival (RFS) was defined as the time interval between the date of surgery and the date of diagnosis of any type of recurrence [26]. To investigate the prognostic role of SNRPD1, we performed IHC using 154 HCC specimens collected from HCC patients who underwent hepatectomy from January 2012 to December 2013 in 900 Hospital of the Joint Logistics Team. We procured the last follow-up on December 31，2018. Child-Pugh classification and the 2010 International Union Against Cancer Tumor-Node-Metastasis (TNM) classification system was used to evaluate the liver function and tumor stages, respectively [27, 28]. The including criteria of patients are the following: only one tumor node and no metastasis, Child-Pugh class A, no cancer radiotherapy or chemotherapy history before the operation, postoperative pathology confirmed as HCC. Patients younger than 18 years old, who died from non-tumor causes within 1 week after surgery and underwent repeat hepatectomy were excluded from our study. We obtained clinicopathological data of patients from the medical record system of the hospital. Survival information was acquired from the follow-up record and the Social Security Death Index. This study was performed according to the relevant medical ethics regulations and approved by the Human Research Ethics Committee of 900 Hospital of the Joint Logistics Team (Fuzhou, China). All participants gave written informed consent prior to surgery and collection of 
the specimens.

\subsection{Analysis of Immunohistochemical staining.}

154 paraffin-embedded with HCC tissue samples were cut into $4 \mu \mathrm{m}$ sections, and then fixed them on microscope slides. The tissue sections were deparaffinized with different concentrations of malondialdehyde and rehydrated with ethanol. Antigen retrieval was performed by boiling the slices in sodium citrate buffer ( $\mathrm{pH}$ 6.0) for 30 minutes. The sections were incubated in $3 \% \mathrm{H}_{2} \mathrm{O}_{2}$ for 10 minutes to inhibit endogenous peroxidase and then washed 3 times in phosphate-buffered saline (PBS). Next, polyclonal rabbit anti-SNRPD1 antibody (1:100; ab233115, UK) was added dropwise to the slices, incubated at $4^{\circ} \mathrm{C}$ overnight, and washed 3 times in PBS. Then, the sections were incubated with secondary antibodies (1:50,000; KIT-5010; anti-rabbit/ mouse IgG; China Fuzhou Maixin Biotechnology Development Co., Ltd.) for 30 minutes at room temperature. Next, the sections were stained with 3,3'-diaminobenzidine and substrate chromogen (Dako) for 2 minutes at room temperature and counterstained with hematoxylin for 40 seconds. The negative control group used PBS instead of the anti-SNRPD1 antibody. CX41 microscope (Olympus, Japan) was used to view the IHC staining. The positive IHC staining was independently evaluated by two independent pathologists who did not have any patient information in advance. According to the human protein atlas database, the protein expression of SNRPD1 was mainly detected in the nucleus. We used a semi-quantitative scoring system to evaluate the protein expression of SNRPD1. When no positive cells were detected, scored $0 ;<=10 \%$ positive cells, scored 1; $11 \%-25 \%$ positive cells, scored 2; and when $26 \%-50 \%$ positive cells were detected, scored 3 , $51 \%-75 \%$ positive cells, scored 4 , more than $75 \%$ positive cells, scored 5 . A score of 0,1 , and 2 indicates low SNRPD1 expression, whereas a higher score indicates high expression.

\subsection{GO and KEGG analysis and PPI network construction.}

We analyzed the co-expressed genes of SNRPD1 in the cBioportal database and the LinkedOmics (http://www.linkedomics.org/login.php) [29], respectively. Then, the overlapping co-expressed genes analyzed by two databases with Pearson's value greater than 0.6 were identified as SNRPD1 co-expressed genes. Next, we explored the gene function of SNRPD1 by performed GO and KEGG enrichment analysis on overlapping SNRPD1 co-expressed genes using the Functional Annotation
Tool in the Database for Annotation, Visualization and Integrated Discovery (DAVID, https:// david.ncifcrf.gov/) database [30]. The protein-protein interaction (PPI) network was constructed in the STRING database (https://www.string-db.org/) with the minimum required interaction score was set as 0.9 (highest confidence) [31]. Finally, the PPI network was visualized in Cytoscape software.

\subsection{GSEA}

The normalized gene expression RNAseq data was downloaded from the UCSC Xena database [32]. The 374 HCC specimens were divided into high expression groups and low expression groups with the median of SNRPD1 expression as the critical point. Then, the enrichment analysis was performed on these expressed data using GSEA software (V4.1.0) [33]. In this process, the KEGG gene sets (c2.cp.kegg.v7.0.symbols.gmt) was selected as the functional gene set, other parameters as the default settings. The pathway of gene enrichment with a normal p-value $<0.05$ and FDR $q$-value $<0.25$ has the significance of the statistics.

\subsection{Analysis of miRNAs related to SNRPD 1 expression in HCC.}

We queried the miRNAs associated with SNRPD1 expression in the "cBioPortal", "LinkedOmics", "MIRWork", and "TargetScan" databases, respectively, then used Venny software to screen out the overlapping miRNAs. Next, we analyzed the correlations between overlapping miRNAs expression and the clinicopathological outcomes in the LinkedOmics database. We also performed the Kaplan-Meier curve and log-rank test analyses in the Kaplan-Meier plotters database to evaluate the relationship between miRNAs and OS of patients with HCC.

\subsection{Cell culture and plasmid transfection.}

We purchased the human normal hepatocyte cell line LO2 from the Chinese Academy of Sciences Committee Type Culture Collection cell bank (Shanghai, China). The HCC cell lines Huh7 and HepG2 were purchased from the American Type Culture Collection (ATCC, USA). All these cell lines were cultured in DMEM basal medium (Hyclone, SH30022.01) supplemented with $100 \mathrm{U} / \mathrm{mL}$ penicillin-100 $\mathrm{\mu g} / \mathrm{mL}$ streptomycin (Hyclone, SV30010) and 10\% fetal bovine serum (FBS, Gibco, 10099141). The DMEM basal medium was placed in an incubator at $37{ }^{\circ} \mathrm{C}$ with $5 \% \mathrm{CO} 2$ and saturated humidity. Vectors used in cell transfection including siRNA of SNRPD1, siRNA negative control (si-NC), miR-100 mimic, and mimic negative control 
(mimic-NC) and were synthesized by Zolgene (Fuzhou, China).

\subsection{Quantitative polymerase chain reaction (qRT-PCR).}

We used RNAiso Plus (TaKaRa, 9109, China) to extract the total RNA of the cells and then used gDNA Purge (Novoprotein, E047-01A, China) to synthesize cDNA based on the manufacturer's instruction. Then, the qPCR was performed to calculate the mRNA expression of SNRPD1 and miR-100 with NovoStart ${ }^{\circledR}$ SYBR qPCR SuperMix Plus (Novoprotein, E096-01B, China) and 7300 Real-Time PCR System (Applied Biosystems, USA). GAPDH and U6 were used as internal controls for SNRPD1 and miR-100, respectively. The primers for GAPDH, U6, SNRPD1, and miR-100 were synthesized by SunYa (Fuzhou, China). The relative expression of SNRPD1 and miR-100 mRNA was determined by using the $\mathrm{Ct}$ value and the $2^{-\Delta \Delta \mathrm{Ct}}$ method.

\subsection{Luciferase reporter assay.}

We used luciferase reporter assay to validate the interaction between SNRPD1 and miR-100. The wild-type (WT) and mutant (MUT) SNRPD1 3'-UTR were designed and inserted into psicheck2.0 vectors (Zolgene, ZVE2012, China). Subsequently, HepG2 cells were co-transfected by combined psicheck2.0 vectors with miR-100 mimic or mimic NC using Lipofectamine 3000 (Invitrogen, Carlsbad, CA, USA). The HCC cells transfected by empty vectors defined as the mock group. Then, the dual-luciferase assays were carried out after incubation for $48 \mathrm{~h}$ to detect the luciferase activity in transfected cells using Duo-LiteTM Luciferase Assay System (Vazyme Biotech Co.,Ltd, China) according to the manufacturer's recommendations.

\subsection{Western blot analysis.}

We lysed the HepG2 cells transfected with si-SNRPD1 or si-NC using RIPA buffer (Meilunbio, China) and incubated on ice for 30mins then extracted the total protein. We separated the total protein by $10 \%$ SDS-PAGE (Beyotime, China), then transferred the separated protein to PVDF membrane (Millipore, USA), and then blocked it in 5\% non-fat skimmed milk powder (BBI, China) at room temperature for 2 hours. Next, we incubated the membranes with the primary antibodies (SNRPD1: Abcam, ab233115, UK; mTOR: Proteintech, 66888-1-Ig, China; LC3: Abcam, ab192890, UK) overnight at $4^{\circ} \mathrm{C}$. Thereafter, we incubated the membranes with the second antibodies (HRP-conjugated Affinipure Goat Anti-Mouse/ Rabbit IgG: Proteintech, SA00001-1/SA00001-2, China) for $1 \mathrm{~h}$ at $25{ }^{\circ} \mathrm{C}$. Finally, we used the chemiluminescence imaging system (BIO-RAD, USA) to detect the protein blots, and Image Lab software (BIO-RAD, USA) was used to measure the protein expression value.

\subsection{Autophagy analysis by transmission electron microscopy (TEM).}

After transfecting HepG2 cells with siRNA or si-NC for 48 hours, the cells were fixed in 5\% glutaraldehyde and $0.1 \mathrm{~mol} / \mathrm{L}$ phosphate buffer $(\mathrm{pH}$ 7.4) for 2 hours at room temperature. The samples were washed three times with PBS buffer and then fixed with $1 \%$ osmium tetroxide and $0.1 \mathrm{~mol} / \mathrm{L}$ phosphate buffer ( $\mathrm{pH}$ 7.4) for $1.5 \mathrm{~h}$. Next, the cells were rinsed with distilled water, dehydrated in different concentrations of ethanol, and embedded in epoxy resin. The embedded samples were cut into ultrathin sections with a thickness of $70 \mathrm{~nm}$ and then stained with uranyl acetate and lead citrate for 5 mins. We used transmission electron microscopy (HT7700, HITACHI, Tokyo, Japan) to observe ultrastructures of cells undergoing autophagy and obtain the images.

\subsection{Statistical analysis.}

Pearson's chi-square test was utilized to compare the categorical variables. The student's t-test was used to compare the normally distributed continuous variables. OS and RFS were analyzed using KaplanMeier plots with the log-rank test. ROC curve with AUC was utilized to evaluate the diagnostic significance of SNRPD1 and miR-100 for HCC. All assays were repeated at least 3 times. The statistical analysis was carried out using Stata Statistical Software: SPSS 19 (SPSS Inc., Chicago, IL, USA) and GraphPad Prism 5.0 (GraphPad Software, Inc., San Diego, CA, USA). P<0.05 represented a statistically significant difference unless otherwise stated.

\section{Results}

\subsection{The high SNRPDI expression is associated with poor survival in HCC patients in the TCGA database.}

The median mRNA expression of SNRPD1 distributed in the tumor tissues in the interactive bodymap is higher than normal tissues (Fig. 1A). Besides, we analyzed the expression of SNRPD1 in a variety of human tumors and paired normal tissues in the TCGA and TNMplot database (Fig. 1B-C). The analysis of CCLE exhibited that 29 hepatocellular carcinoma lines have the copy number variation of SNRPD1 mRNA expression (Fig. 1D). We analyzed the expression of SNRPD1 in HCC tissues and normal tissues in the GEPIA database. The results showed that the expression of SNRPD1 in HCC tissues was significantly higher than normal liver tissues (Fig. 2A), and incrementally upregulated with increasing 
tumor stages (Fig. 2B). We performed the KaplanMeier analysis in the GEPIA database and results demonstrated that high SNRPD1 expression in patients with HCC correlated with shorter overall survival $(\mathrm{HR}=2, \mathrm{P}<0.001$; Fig. $2 \mathrm{C})$ and disease-free survival ( $\mathrm{HR}=1.4, \mathrm{P}=0.016$; Fig. $2 \mathrm{D})$.

\subsection{The relationship between the SNRPDI expression and clinical outcomes in patients with HCC in the GEO database.}

We downloaded the GSE14520 dataset from the GEO database to analyze the relationship between SNRPD1 expression and the clinical outcomes of HCC patients. The mRNA expression of SNRPD1 in the HCC tissues $(n=247)$ was significantly higher than the non-HCC tissues $(\mathrm{n}=241, \mathrm{P}<0.001)$ (Fig. 2E). The high SNRPD1 mRNA expression was significantly positively correlated to the TNM stage $(\mathrm{P}=0.025)$, serum AFP level $(\mathrm{P}<0.001)$, CLIP staging $(\mathrm{P}<0.001)$. Gender, age, tumor size, ALT, BCLC staging, multinodular, and cirrhosis were not correlated to the SNRPD1 expression (Table 1). We found that Tumor size $(\mathrm{P}=0.001)$, TNM staging $(\mathrm{P}<0.001)$, Serum AFP level $(\mathrm{P}=0.011), \mathrm{BCLC}$ staging $(\mathrm{P}<0.001)$, $\mathrm{CLIP}$ staging

\section{A}

Interactive Bodymap

The median SNRPD1 expression of normal and tumor samples in bodymap
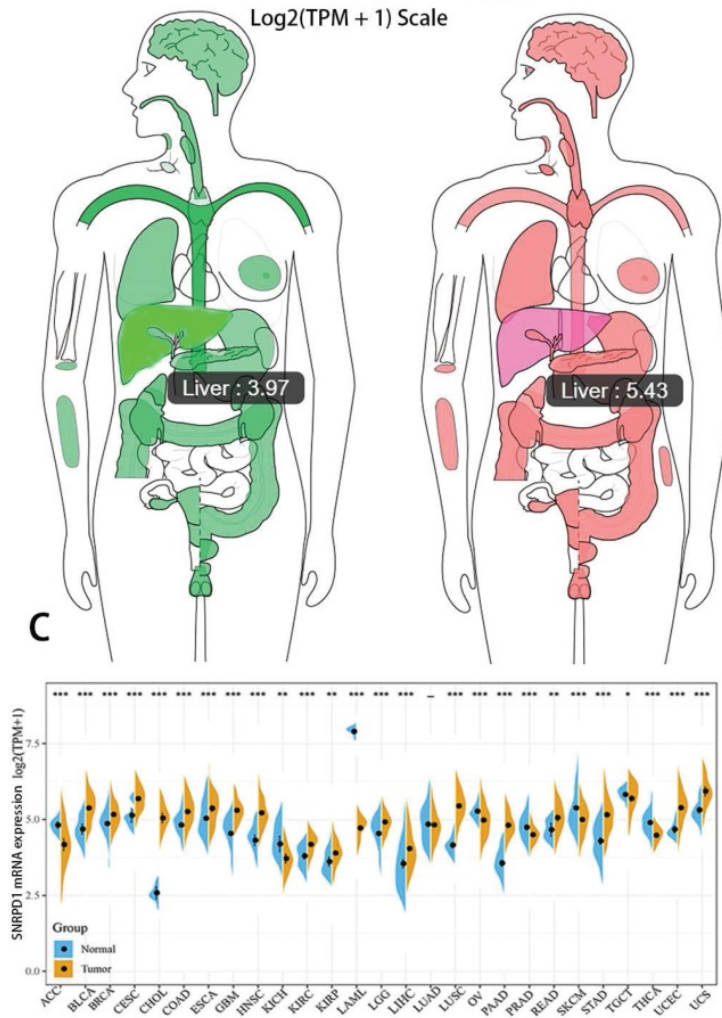

$(\mathrm{P}=0.001), \quad$ Multinodular $\quad(\mathrm{P}=0.023), \quad$ Cirrhosis $(\mathrm{P}=0.023)$, and high SNRPD1 expression $(\mathrm{P}=0.003)$ were risk factors for overall survival of HCC by performing Univariate Cox Regression analysis. The Multivariate Cox Regression analysis confirmed that BCLC staging (HR (95\%CI): 5.381(2.985-9.700); $\mathrm{P}<0.001)$, Multinodular $\quad(\mathrm{HR} \quad(95 \% \mathrm{CI})$ : 2.012(1.082-3.740); $\mathrm{P}=0.027)$, Cirrhosis (HR (95\% CI): $0.206(0.050-0.839) ; \quad \mathrm{P}=0.028)$ and high SNRPD1 expression (HR (95\%CI): 1.968(1.266-3.060); $\mathrm{P}=0.003$ ) were independent risk factors for overall survival. For RFS, the Gender $(\mathrm{P}=0.009)$, TNM staging $(\mathrm{P}<0.001)$, BCLC staging $(\mathrm{P}<0.001)$, CLIP staging $(\mathrm{P}=0.016)$, and high SNRPD1 expression $(\mathrm{P}=0.034)$ were risk factors. Meanwhile, Gender (HR (95\%CI): 0.516(0.269-0.990); $\mathrm{P}=0.047)$, BCLC staging (HR (95\% CI): 2.496(1.697-3.672); $\quad \mathrm{P}<0.001)$ and high SNRPD1 expression (HR (95\%CI): 1.299(0.878-1.920); $\mathrm{P}=0.042$ ) were independent risk factors of RFS analyzed by Multivariate Cox Regression (Table 2). The Kaplan-Meier analysis and log-rank test analysis demonstrated that high SNRPD1 mRNA expression led to a poor OS ( $p=0.0022$, Fig. $2 F)$ and RFS $(P=0.033$, Fig $2 \mathrm{G}$ ) in patients with HCC. The receiver operating

\section{B}
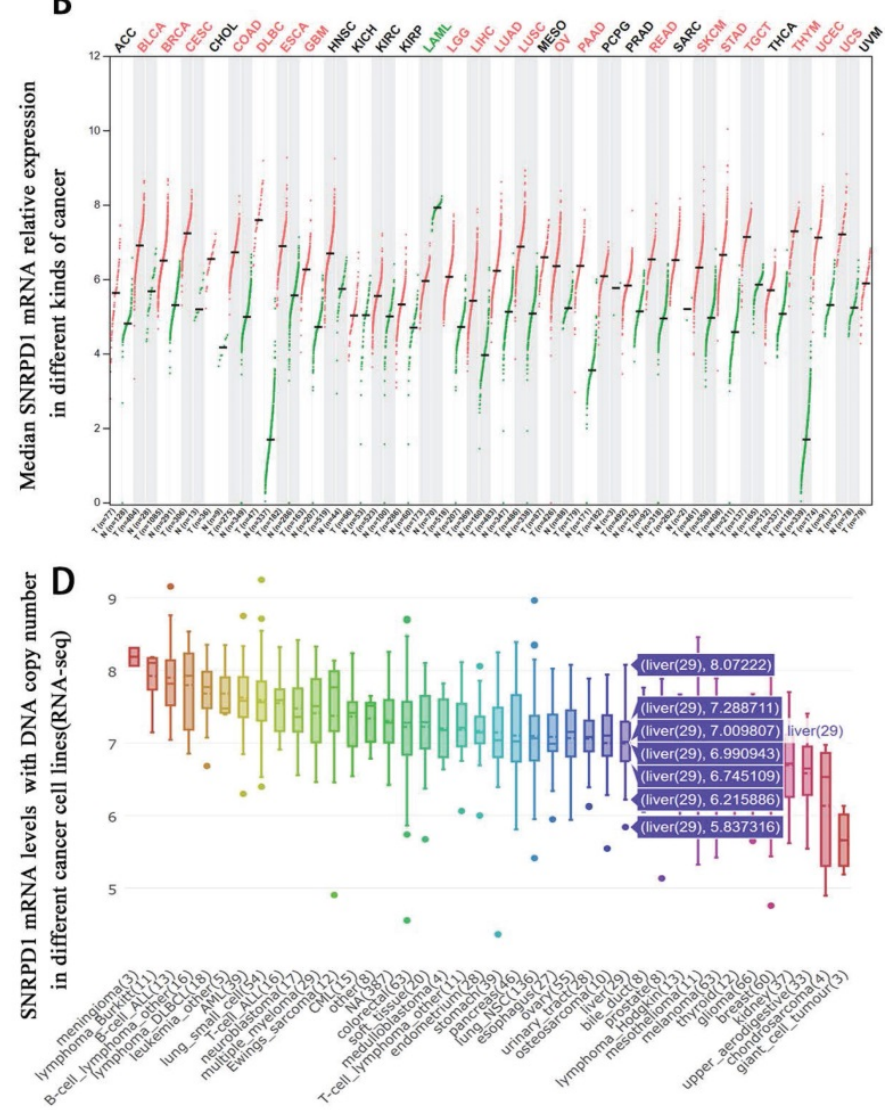

Figure 1. The mRNA expression level of SNRPD1 in various kinds of tumor samples and paired normal tissues. (A) The median SNRPD1 expression of HCC (red, 5.43) and normal liver samples (green, 3.97) in bodymap in the GEPIA. (B) The SNRPDI expression profile across various kinds of tumor samples (red) and paired normal samples (green) in the GEPIA. Each dots represent the expressed value of each sample. (C)The SNRPDI expression profile across all tumor samples and paired normal samples in TNMplot database. (D) The SNRPDI mRNA levels with DNA copy number expressed in different kinds of cancer cell lines in the Cancer Cell Line Encyclopedia (CCLE) database. 29 HCC cell lines have variations in SNRPDI expression. 
characteristic (ROC) curve revealed that SNRPD1 expression has a significant diagnosis value on HCC (AUC $=0.819, \mathrm{P}<0.001$, Fig. $2 \mathrm{H}$ ). In addition, SNRPD1 mRNA expression incrementally upregulated with normal, tumor, and metastatic tissues analyzed in the
TNMplot database ( $p=1.28 \mathrm{e}-49$, Fig. 2I). Furthermore, the analysis of SNRPD1 protein expression in the Human Protein Atlas database showed that its protein level in HCC samples (Fig. 2K-L) was higher than normal liver tissues (Fig. 2J).
A

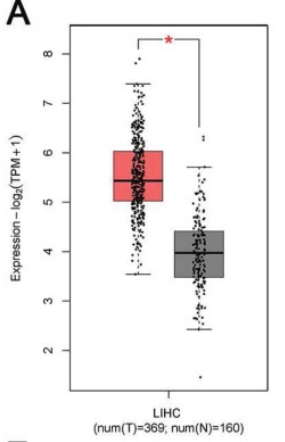

E
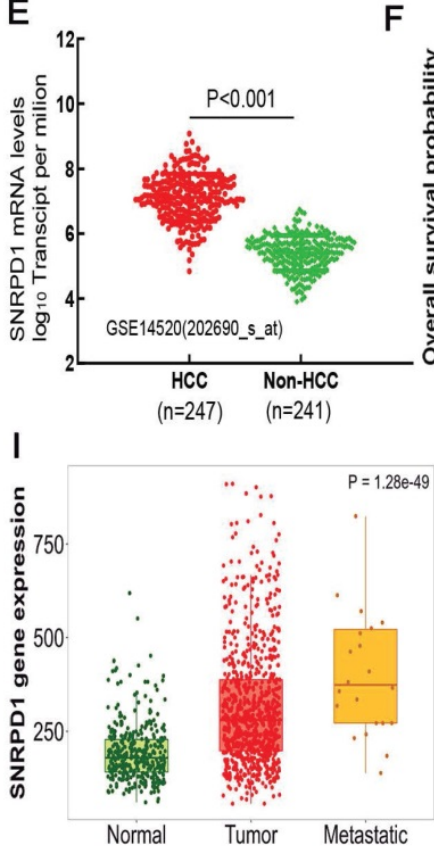

B

$F$
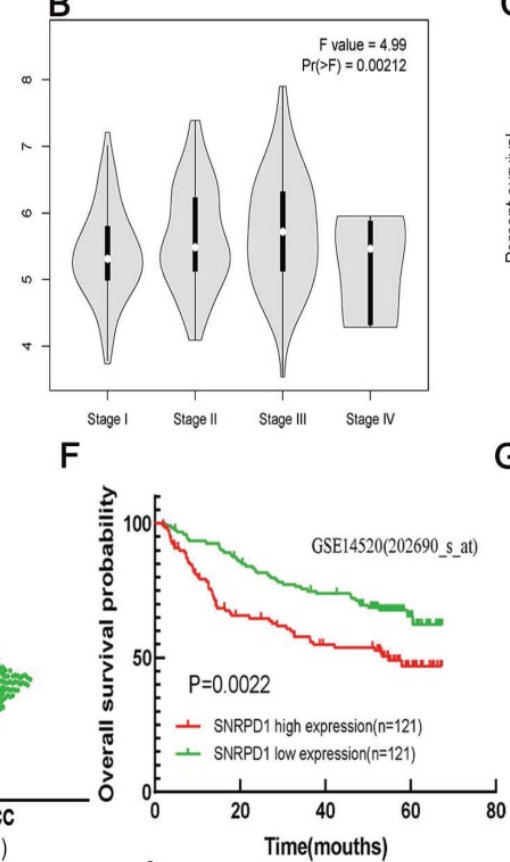

$J$

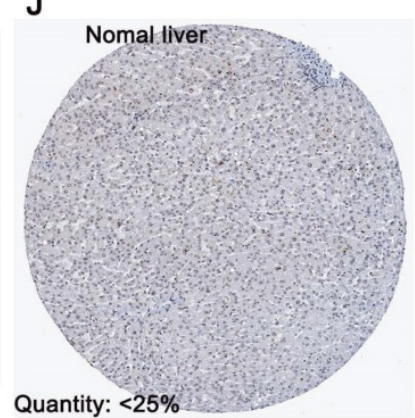

C

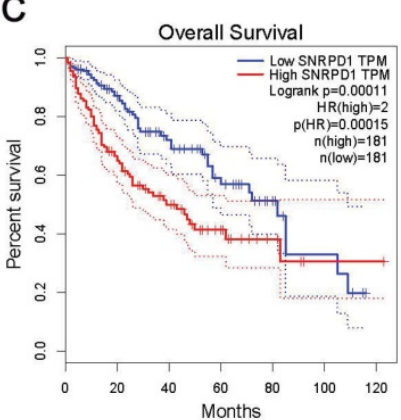

G

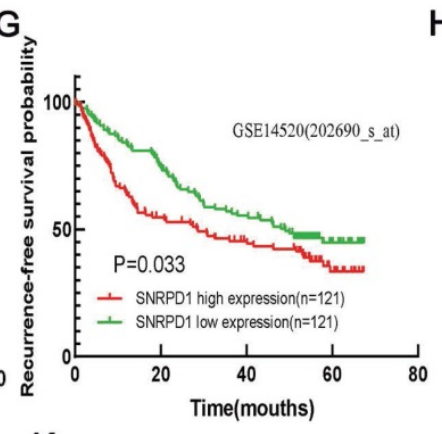

$\mathrm{K}$

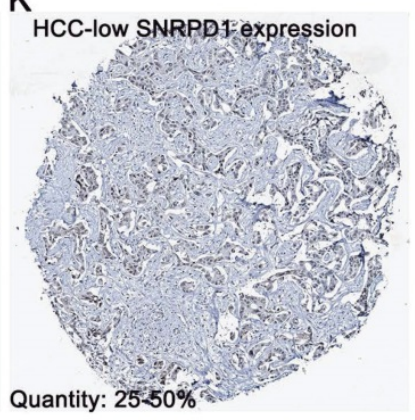

D Disease Free Survival

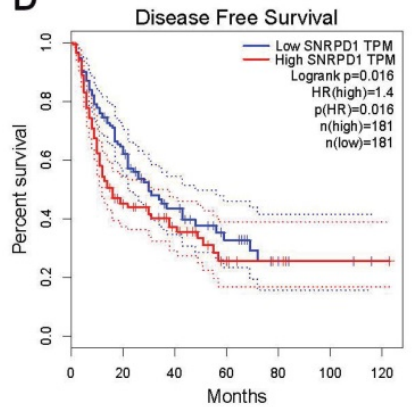

$\mathrm{H}$

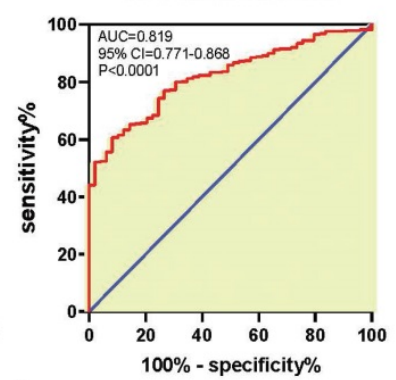

L

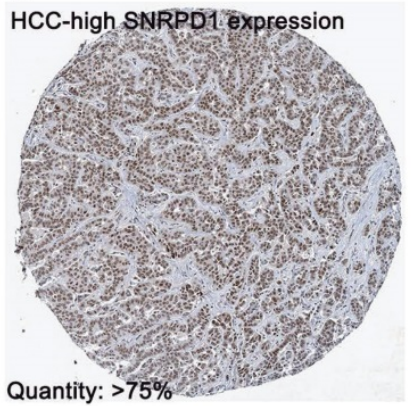

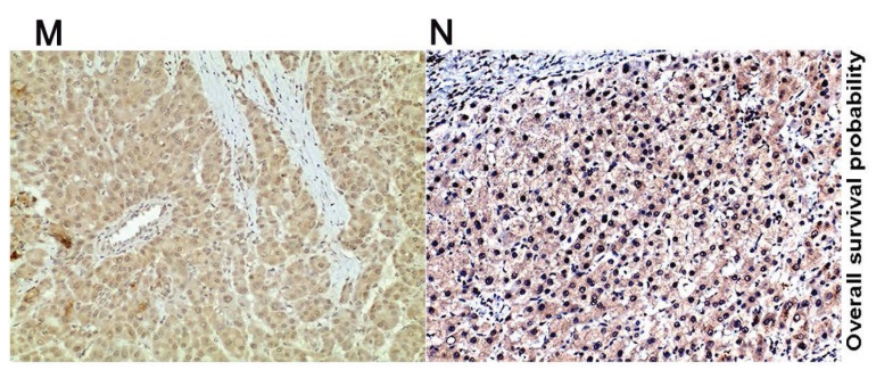
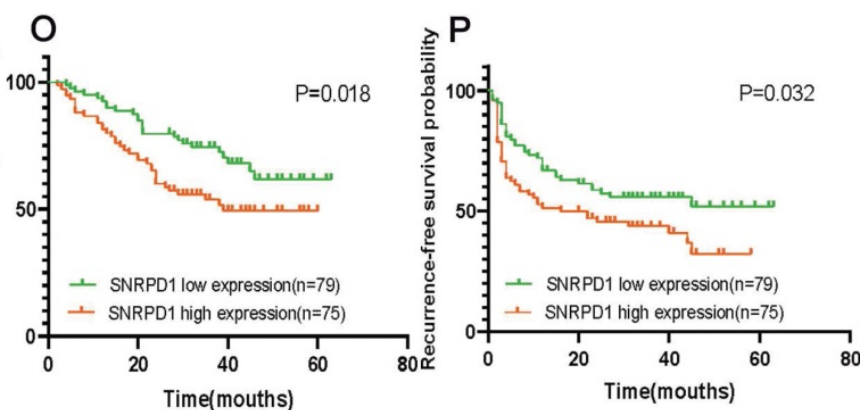

Figure 2. High mRNA and protein expression of SNRPDI correlates with poor prognosis in HCC patients. (A) The SNRPDI expression level in HCC samples was significantly higher than normal liver tissues in the GEPIA database $(P<0.05)$. (B) The SNRPDI expression in HCC samples was incrementally upregulated with increasing tumor stages in the GEPIA database. (C-D) High SNRPDI mRNA expression correlates with poor overall survival (C) and diseases free survival (D) of HCC patients in the GEPIA database. (E) SNRPDI expression in HCC tissues was significantly higher than adjacent normal liver tissues in the GSE14520 dataset. (F-G) High SNRPDI mRNA expression correlates with poor overall survival $(F)$ and recurrence-free survival $(G)$ of HCC patients in the GSE14520 dataset. $(H)$ The receiver operating characteristic (ROC) curve revealed that SNRPDI expression has a significant diagnosis value on HCC (AUC $=0.819, \mathrm{P}<0.001)$. (I) The SNRPDI mRNA expression was incrementally upregulated in the normal, tumor, and metastatic tissues of HCC patients in the TNMplot database (https://www.tnmplot.com/). (J-L) Representative images of immunohistochemical (IHC) staining of SNRPDI protein expression in normal liver tissues (l, expression quantity $<25 \%$ ), low expression $\mathrm{HCC}$ tissues (K, expression quantity $25-50 \%$ ), and high expression $\mathrm{HCC}$ tissues in the Human Protein Atlas database (L, expression quantity $>75 \%$ ). (M-N) Representative image of IHC staining of SNRPDI low (M)/high (N) protein expression in tumor tissue from 154 patients with HCC (x200 magnification). (O-P) High SNRPD1 protein expression correlates with poor overall survival (O) and diseases free survival (P) of 154 HCC patients. 
Table 1. Correlation between SNRPD1 expression and clinical outcomes in HCC patients (242 cases, GSE14520 dataset).

\begin{tabular}{|c|c|c|c|c|c|}
\hline \multirow[t]{2}{*}{ Characteristics } & & \multicolumn{3}{|c|}{ SNRPD1 level } & \multirow[b]{2}{*}{${ }^{*} P$-Value } \\
\hline & & $\mathrm{N}$ & $\operatorname{high}(\mathrm{n})$ & $\operatorname{low}(\mathrm{n})$ & \\
\hline \multirow[t]{2}{*}{ Gender } & Male & 211 & 103 & 108 & 0.336 \\
\hline & Female & 31 & 18 & 13 & \\
\hline \multirow[t]{2}{*}{ Age } & $>55$ & 75 & 32 & 43 & 0.126 \\
\hline & $<=55$ & 167 & 89 & 78 & \\
\hline \multirow[t]{2}{*}{ Tumor size } & $>5 \mathrm{~cm}$ & 88 & 46 & 42 & 0.559 \\
\hline & $<=5 \mathrm{~cm}$ & 153 & 74 & 79 & \\
\hline \multirow[t]{2}{*}{ TNM stage } & $\mathrm{I} / \mathrm{II}$ & 176 & 83 & 93 & 0.025 \\
\hline & III & 49 & 32 & 17 & \\
\hline \multirow[t]{2}{*}{ Serum AFP level } & $>300 \mathrm{ng} / \mathrm{ml}$ & 110 & 73 & 37 & $<0.001$ \\
\hline & $<=300 \mathrm{ng} / \mathrm{ml}$ & 128 & 47 & 81 & \\
\hline \multirow[t]{2}{*}{ ALT } & $>50 \mathrm{U} / \mathrm{L}$ & 100 & 49 & 51 & 0.794 \\
\hline & $<=50 \mathrm{U} / \mathrm{L}$ & 142 & 72 & 70 & \\
\hline \multirow[t]{3}{*}{ BCLC staging } & Yes & 225 & 115 & 110 & 0.746 \\
\hline & $0-\mathrm{A}$ & 173 & 86 & 87 & \\
\hline & $\mathrm{B}-\mathrm{C}$ & 52 & 29 & 23 & \\
\hline \multirow[t]{2}{*}{ CLIP staging } & 0 & 98 & 36 & 62 & $<0.001$ \\
\hline & $1-5$ & 127 & 79 & 48 & \\
\hline \multirow[t]{2}{*}{ Multinodular } & Yes & 51 & 29 & 22 & 0.270 \\
\hline & No & 191 & 92 & 99 & \\
\hline \multirow[t]{2}{*}{ Cirrhosis } & Yes & 19 & 12 & 7 & 0.232 \\
\hline & No & 223 & 109 & 114 & \\
\hline
\end{tabular}

Abbreviations: AFP - alpha fetoprotein, TNM - tumor, node, metastasis; ALT, alanine aminotransferase. BCLC, Barcelona Clinic Liver Cancer. ${ }^{*} P$-Value $<0.05$ were considered statistically significant.

\subsection{The relationship between the expression of SNRPDI and clinicopathologic characteristics in $154 \mathrm{HCC}$ patients.}

As shown in the Figures, our IHC validated that SNRPD1 mainly expressed on the nucleus of HCC cells (Fig. 2M-N). Based on the semi-quantitative scoring system, we divided 154 cases of HCC into high expression groups $(\mathrm{n}=75)$ and low expression groups $(n=79)$. The SNRPD1 protein expression level was significantly correlated to Age $(\mathrm{P}=0.049)$, TNM staging $(\mathrm{P}=0.010)$, Serum AFP level $(\mathrm{P}=0.001)$, Tumor differentiation $(\mathrm{P}=0.002)$, Vascular invasion $(\mathrm{P}=0.006)$, Recurrence $(\mathrm{P}=0.036)$, and Survival $(\mathrm{P}=0.025)$ (Table $3)$. Univariate Cox Regression analysis showed that TNM staging $(\mathrm{P}=0.014)$, Vascular invasion $(\mathrm{P}=0.033)$, and high SNRPD1 protein expression $(\mathrm{P}=0.015)$ were risk factors for OS of HCC. The Multivariate Cox Regression analysis confirmed that TNM staging (HR (95\%CI): $1.997(1.136-3.511) ; \quad \mathrm{P}=0.016)$ and high SNRPD1 expression (HR (95\%CI): 1.890(1.098-3.255); $\mathrm{P}=0.022)$ were independent risk factors for OS. For RFS of HCC, TNM staging $(\mathrm{P}=0.027)$, Tumor differentiation $(\mathrm{P}=0.046)$, Vascular invasion $(\mathrm{P}=0.002)$, Tumor encapsulation $(\mathrm{P}<0.001)$, and high SNRPD1 expression $(\mathrm{P}=0.030)$ were risk factors. Meanwhile, the multivariate cox regression revealed that TNM staging (HR (95\% CI): 1.682(1.049-2.699); $\mathrm{P}=0.031)$, Vascular invasion (HR (95\% CI): 1.861(1.122-3.087); $\mathrm{P}=0.016)$, Tumor encapsulation (HR $(95 \% \mathrm{CI})$ : 0.209(0.129-0.338); $\mathrm{P}<0.001)$, and high SNRPD1 protein expression (HR (95\% CI): 1.735(1.070-2.813); $\mathrm{P}=0.026$ ) were independent risk factors (Table 4). Finally, The Kaplan-Meier analysis showed that high SNRPD1 protein expression led to a poor OS $(\mathrm{p}=0.018$, Fig. $2 \mathrm{O})$ and RFS $(\mathrm{P}=0.032$, Fig $2 \mathrm{P})$ in patients with HCC.

Table 2. Univariate and Multivariate Cox Regression analysis of overall survival and Recurrence-free survival in HCC patients (242 cases, GSE14520 dataset).

\begin{tabular}{|c|c|c|c|c|c|}
\hline \multirow[t]{2}{*}{ Variables } & & \multirow{2}{*}{$\begin{array}{l}\text { Overall survival } \\
\text { HR }(95 \% \mathrm{CI})\end{array}$} & \multirow[t]{2}{*}{${ }^{*} P$-Value } & \multirow{2}{*}{$\frac{\text { Recurrence-free survival }}{\mathrm{HR}(95 \% \mathrm{CI})}$} & \multirow[t]{2}{*}{${ }^{*} P$-Value } \\
\hline & & & & & \\
\hline \multicolumn{6}{|l|}{ Univariate analysis } \\
\hline Gender & Male vs. female & $0.538(0.261-1.110)$ & 0.094 & $0.424(0.223-0.807)$ & 0.009 \\
\hline Age(years) & $>55$ vs. $<=55$ & $0.759(0.484-1.191)$ & 0.231 & $1.022(0.714-1.464)$ & 0.905 \\
\hline Tumor size $(\mathrm{cm})$ & $>5$ vs. $<=5$ & $1.953(1.303-2.928)$ & 0.001 & $0.713(0.504-1.008)$ & 0.055 \\
\hline TNM staging & I/II vs. III & $0.275(0.177-0.428)$ & $<0.001$ & $2.215(1.499-3.274)$ & $<0.001$ \\
\hline Serum AFP level(ng/ml) & $>300$ vs $<=300$ & $1.686(1.126-2.527)$ & 0.011 & $0.761(0.543-1.067)$ & 0.113 \\
\hline $\operatorname{ALT}(\mathrm{U} / \mathrm{L})$ & $>50$ vs $<=50$ & $0.866(0.579-1.295)$ & 0.483 & $0.724(0.517-1.014)$ & 0.060 \\
\hline BCLC staging & 0 -A vs. B-C & $3.692(2.381-5.726)$ & $<0.001$ & $2.647(1.803-3.888)$ & $<0.001$ \\
\hline CLIP staging & 0 vs $1-5$ & $2.194(1.384-3.478)$ & 0.001 & $1.577(1.085-2.234)$ & 0.016 \\
\hline Multinodular & Yes vs. no & $0.599(0.385-0.931)$ & 0.023 & $0.734(0.495-1.088)$ & 0.124 \\
\hline Cirrhosis & Yes vs. no & $5.093(1.255-20.671)$ & 0.023 & $0.499(0.233-1.068)$ & 0.074 \\
\hline SNRPD1 & High vs. low & $0.535(0.356-0.803)$ & 0.003 & $0.695(0.496-0.973)$ & 0.034 \\
\hline \multicolumn{6}{|l|}{ Multivariate analysis } \\
\hline Gender & Male vs. female & & & $0.516(0.269-0.990)$ & 0.047 \\
\hline Age(years) & $>55$ vs. $<=55$ & & & & \\
\hline Tumor size $(\mathrm{cm})$ & $>5$ vs. $<=5$ & $0.945(0.517-1.727)$ & 0.544 & & \\
\hline TNM staging & I/II vs. III & $1.362(0.625-2.971)$ & 0.322 & $0.976(0.524-1.816)$ & 0.780 \\
\hline Serum AFP level(ng/ml) & $>300$ vs $<=300$ & $1.366(0.695-2.686)$ & 0.741 & & \\
\hline $\operatorname{ALT}(\mathrm{U} / \mathrm{L})$ & $>50$ vs $<=50$ & & & & \\
\hline BCLC staging & 0-A vs. B-C & $5.381(2.985-9.700)$ & $<0.001$ & $2.496(1.697-3.672)$ & $<0.001$ \\
\hline CLIP staging & 0 vs $1-5$ & $1.751(0.787-3.894)$ & 0.274 & $1.136(0.741-1.742)$ & 0.330 \\
\hline Multinodular & Yes vs. no & $2.012(1.082-3.740)$ & 0.027 & & \\
\hline Cirrhosis & Yes vs. no & $0.206(0.050-0.839)$ & 0.028 & & \\
\hline SNRPD1 & High vs. low & $1.968(1.266-3.060)$ & 0.003 & $1.299(0.878-1.920)$ & 0.042 \\
\hline
\end{tabular}

Abbreviations: HR, hazard ratio; CI, confidential interval; AFP - alpha fetoprotein, TNM - tumor, node, metastasis; ALT, alanine aminotransferase. BCLC, Barcelona Clinic Liver Cancer. ${ }^{*} P$-Value $<0.05$ were considered statistically significant. 
Table 3. Correlation between SNRPDI expression and clinical outcomes in HCC patients $(n=154)$.

\begin{tabular}{|c|c|c|c|c|c|c|}
\hline \multicolumn{2}{|l|}{ Characteristics } & \multirow[t]{2}{*}{$\mathrm{N}$} & \multicolumn{2}{|c|}{ SNRPD1 level } & \multirow[t]{2}{*}{$X^{2}$} & \multirow[t]{2}{*}{${ }^{*} P$-Value } \\
\hline & & & high(n) & $\operatorname{low}(\mathrm{n})$ & & \\
\hline \multirow[t]{2}{*}{ Age (year) } & $>55$ & 101 & 55 & 46 & 3.889 & 0.049 \\
\hline & $<=55$ & 53 & 20 & 33 & & \\
\hline \multirow[t]{2}{*}{ Gender } & Male & 134 & 66 & 68 & 0.126 & 0.723 \\
\hline & Female & 20 & 9 & 11 & & \\
\hline \multirow[t]{2}{*}{ Tumor size $(\mathrm{cm})$} & $>5 \mathrm{~cm}$ & 82 & 37 & 45 & 0.889 & 0.343 \\
\hline & $<=5 \mathrm{~cm}$ & 72 & 38 & 34 & & \\
\hline \multirow[t]{2}{*}{ TNM staging } & $\mathrm{I} / \mathrm{II}$ & 103 & 39 & 57 & 6.655 & 0.010 \\
\hline & III & 51 & 36 & 22 & & \\
\hline \multirow[t]{2}{*}{ Serum AFP level } & $>400 \mathrm{ng} / \mathrm{ml}$ & 81 & 50 & 31 & 11.607 & 0.001 \\
\hline & $<=400 \mathrm{ng} / \mathrm{ml}$ & 73 & 25 & 48 & & \\
\hline \multirow[t]{2}{*}{ Tumor location } & Left & 52 & 21 & 31 & 2.174 & 0.140 \\
\hline & Right & 102 & 54 & 48 & & \\
\hline \multirow[t]{3}{*}{ Tumor differentiation } & Low & 20 & 14 & 6 & 12.472 & 0.002 \\
\hline & Median & 98 & 52 & 46 & & \\
\hline & High & 36 & 9 & 27 & & \\
\hline \multirow[t]{2}{*}{ Vascular invasion } & Yes & 73 & 44 & 29 & 7.440 & 0.006 \\
\hline & No & 81 & 31 & 50 & & \\
\hline \multirow[t]{2}{*}{ Tumor encapsulation } & Yes & 104 & 50 & 54 & 0.050 & 0.823 \\
\hline & No & 50 & 25 & 25 & & \\
\hline \multirow[t]{2}{*}{ HBV DNA load } & $>10^{4}$ & 72 & 38 & 33 & 1.225 & 0.268 \\
\hline & $<=10^{4}$ & 82 & 37 & 46 & & \\
\hline \multirow[t]{2}{*}{ Recurrence } & Yes & 78 & 44 & 34 & 4.393 & 0.036 \\
\hline & No & 76 & 31 & 45 & & \\
\hline \multirow[t]{2}{*}{ Survival } & Alive & 94 & 39 & 55 & 5.023 & 0.025 \\
\hline & Dead & 60 & 36 & 24 & & \\
\hline
\end{tabular}

Abbreviations: AFP - alpha fetoprotein, TNM tumor, node, metastasis. ${ }^{*} P$-Value $<0.05$ were considered statistically significant.

\subsection{SNRPDI alterations analysis in TCGA database and gene function analysis through GO, KEGG, and GSEA.}

We queried the SNRPD1 alterations in a cohort of 347 HCC patients (TCGA, PanCancer Atlas) in the cBioportal database. The result showed that SNRPD1 altered in $25(7 \%)$ of queried HCC patients, including 2 cases of amplification, and 23 cases of mRNA high expression (Fig. 3A). In addition, the Kaplan-Meier curve shows that the OS ( $\mathrm{P}=0.034$, Fig. $3 \mathrm{~B})$, as well as the disease-free survival $(\mathrm{P}=0.024$, Fig. $3 \mathrm{C})$ of HCC patients with SNRPD1 alterations $(n=25)$, was poorer than without SNRPD1 alterations $(n=322)$.

We analyzed the co-expressed genes of SNRPD1 using the HCC dataset in the cBioportal database and the LinkedOmics, respectively. 11459 positively and 8627 negatively genes correlated with SNRPD1 protein expression in a cohort of 371 HCC patients from the LinkedOmics database expression were investigated (Fig. 3D). The top 50 positively and negatively correlated genes were exhibited using the heat map, respectively (Fig. 3E-F). 81 overlapping correlated genes with Spearman's Correlation greater than 0.6 obtained in the LinkedOmics and cBioportal database were screened as SNRPD1 co-expressed genes (Fig. 3G). Next, we explored the gene function of SNRPD1 by performing GO and KEGG analysis on
81 SNRPD1 co-expressed genes in the DAVID database. The GO analysis revealed that the SNRPD1 co-expressed genes mainly enriched in Cell division, Nuclear import, mRNA splicing via spliceosome, Mitotic nuclear division, Spliceosomal snRNP assembly, and Regulation of cell cycle, etc. The KEGG analysis revealed that SNRPD1 co-expressed genes were mainly enriched in the signal pathway of Spliceosome, Ribosome, and Cell cycle (Table 5). Then, we explored the significant interactions of SNRPD1 with 81 co-expressed genes using the STRING database, with a confidence score of $>0.900$ (highest confidence). Ultimately, a PPI network with 57 nodes and 212 edges was constructed and visualized in the Cytoscape software. The PPI network showed that the SNRPA, SNRPB, SNRPB2, SNRPD2, SNRPE, SNRPG, POLR2H, and PRMT1 protein can interact with SNRPD1 (Fig. 3H). The Spearman rank correlation test in the cBioPortal database confirmed that SNRPA, SNRPB, SNRPB2, SNRPD2, SNRPE, SNRPG, POLR2H, and PRMT1 protein positively correlated with SNRPD1 (all Spearman $>0.63, \mathrm{P}<0.05$ ) (Fig. 4A). Furthermore, the survival analysis performed in the GEPIA database showed that SNRPA, SNRPB, SNRPB2, SNRPD2, SNRPE, SNRPG, POLR2H, and PRMT1 mRNA expression associated with OS of HCC patients (Fig. 4B).

To further explore gene functions and potential pathways regulating the occurrence and development of HCC, we performed GSEA enrichment analysis using gene expression data downloaded from the TCGA database. The results showed that the KEGG signaling pathways related to occurrence and development of HCC including "ribosome", "base excision repair", "DNA replication", "cell cycle", and "mismatch repair", etc. (Fig. 4C). The GSEA result also showed that the genes enriched in these KEGG pathways were significantly altered in the SNRPD1 aberrantly high expression group (Table 6). In summary, we have the reasonable consideration that SNRPD1 may regulate the tumorigenesis and development of HCC through these signaling pathways.

\section{5. miR-100 as a potential HCC suppressor via negatively targeting SNRPD 1 expression.}

We investigated the 614 positively and 181 negatively correlated microRNAs related to SNRPD1 expression in the LinkedOmics database (Fig. 5A). The top 50 positively and negatively correlated microRNAs were exhibited in the heat map, respectively (Fig. 5B-C). Next, we searched for microRNAs related to SNRPD1 expression in the 
miRWalk, TargetScan, and miRTarBase databases, respectively. Then, we obtained four overlapping microRNAs from the above four databases, namely miR-100, miR-665, miR-940, miR-3911 (Fig. 5D). We then used the Venn Diagrams to intersect the four overlapping microRNAs with the top 50 negatively correlated microRNAs, the remaining one microRNA (miR-100) (Fig. 5E). We studied the expression of miR-100 in the TCGA database and found that the expression of mir-100 in HCC was significantly lower than that in non-HCC tissues $(\mathrm{P}<0.0001$, Fig. 5F). The ROC curve revealed that miR-100 expression has a significant diagnosis value on HCC (AUC $=0.743$, $\mathrm{P}<0.0001$, Fig. 5G). There was a significantly negative correlation between the expression of mir-100 and SNRPD1 in HCC patients ( $\mathrm{r}=-0.4921, \mathrm{P}=3.59 \mathrm{e}-23)$ (Fig. $5 \mathrm{H})$. We also analyzed the relations between the expression of miR-100 and the clinical outcome of HCC patients in the Linkedomics database (Fig. 5I). The results showed that low miR-100 expression was associated with the overall _ survival $(\mathrm{P}=8.925 \mathrm{e}-04$, Fig. 5J), pathology_ $\mathrm{T}_{-}$stage $(\mathrm{P}=1.774 \mathrm{e}-02$, Fig. $5 \mathrm{~K})$, pathologic_ stage $(\mathrm{P}=1.777 \mathrm{e}-02, \quad$ Fig. $5 \mathrm{~L})$, and pathology_ N_Stage $(\mathrm{P}=3.384 \mathrm{e}-02)$, etc.

\subsection{SNRPD 1 is the direct target gene of miR-100 in HCC cells.}

We used one normal liver cell line (LO2) and two HCC cells line (Huh7, HepG2) to investigate the effect of miR-100 on the expression of SNRPD1. The results showed that SNRPD1 (Fig. 6A) expression was significantly upregulated while the expression of miR-100(Fig. 6B) was significantly downregulated in Huh7 and HepG2 cells line when compared with the LO2 cells line (all $\mathrm{P}<0.001$ ). We verified the relations between miR-100 and SNRPD1 expression using qRT-PCR. The results exhibited a significantly upregulated miR-100 expression (Fig. 6C) and a significantly downregulated SNRPD1 expression (Fig. 6D) after the miR-100 mimic was transfected into HepG2 (all $\mathrm{P}<0.001$ ). We further validated the associations between miR-100 with SNRPD1 expression using luciferase reporter assay and found that the HepG2 cells transfected by miR-100 mimic were decreased luciferase activity of WT 3'-UTR of SNRPD1, but mutant SNRPD1-expressing cells and mock group cells showed no luciferase activity decrease (Fig. 6E-F). Overall, these results indicated that SNRPD1 was the direct target gene of miR-100 in HCC and was negatively regulated by miR-100.

\subsection{SNRPDI knockdown leads to the mTOR signaling pathway downregulated in HCC cells.}

Previous studies have found that knockdown the expression of SNRPE, another core SNRP spliceosomal protein, can inhibit the mTOR pathway in breast cancer SKBr-3 cell lines [3]. Therefore, we hypothesized whether SNRPD1 regulates the occurrence and development of HCC through the mTOR pathway. In this present study, we briefly investigated the effect of SNRPD1 mRNA expression on the activity of the mTOR signaling pathway in the HepG2 cell line. As shown in Figure. 7A, the SNRPD1 expression level was successfully inhibited by the siRNA of SNRPD1 (P<0.001, Fig. 7A). The western blot analysis showed that the mTOR protein level of si-SNRPD1 groups was significantly decreased compared with the Mock and si-NC groups $(\mathrm{P}<0.05$, Fig. 7B-C). All these results indicated that mTOR signaling pathway was blocked in HepG2 cells due to low expression of SNRPD1.

Table 4. Univariate and Multivariate Cox Regression analysis of overall survival and Recurrence-free survival in HCC patients ( $n=154)$.

\begin{tabular}{|c|c|c|c|c|c|}
\hline \multirow[t]{2}{*}{ Variables } & & \multirow{2}{*}{$\begin{array}{l}\text { Overall survival } \\
\text { HR }(95 \% \mathrm{CI})\end{array}$} & \multirow[t]{2}{*}{${ }^{*} P$-Value } & \multirow{2}{*}{$\frac{\text { Recurrence-free survival }}{\mathrm{HR}(95 \% \mathrm{CI})}$} & \multirow[t]{2}{*}{${ }^{*} P$-Value } \\
\hline & & & & & \\
\hline \multicolumn{6}{|l|}{ Univariate analysis } \\
\hline Age (year) & $>55$ vs. $<=55$ & $0.825(0.490-1.389)$ & 0.470 & $0.732(0.463-1.158)$ & 0.183 \\
\hline Gender & Male vs. female & $0.779(0.335-1.810)$ & 0.561 & $1.258(0.665-2.381)$ & 0.480 \\
\hline Tumor size $(\mathrm{cm})$ & $>5$ vs. $<=5$ & $1.391(0.705-2.744)$ & 0.341 & $0.978(0.616-1.555)$ & 0.926 \\
\hline TNM staging & I/II vs. III & $1.963(1.144-3.367)$ & 0.014 & $1.677(1.060-2.653)$ & 0.027 \\
\hline Serum AFP level & $>400$ vs $<=400$ & $1.126(0.678-1.868)$ & 0.647 & $1.246(0.799-1.943)$ & 0.332 \\
\hline Tumor location & Left vs. right & $0.757(0.450-1.275)$ & 0.296 & $1.175(0.727-1.901)$ & 0.510 \\
\hline Tumor differentiation & Hihg vs. median/low & $1.613(0.971-2.679)$ & 0.065 & $1.803(1.010-3.220)$ & 0.046 \\
\hline Vascular invasion & Yes vs. no & $1.747(1.045-2.921)$ & 0.033 & $2.075(1.315-3.275)$ & 0.002 \\
\hline Tumor encapsulation & Yes vs. no & $0.788(0.466-1.334)$ & 0.376 & $0.259(0.164-0.488)$ & $<0.001$ \\
\hline HBV DNA load & $>10^{4}$ vs $<=10^{4}$ & $1.395(0.840-2.314)$ & 0.198 & $0.984(0.630-1.538)$ & 0.944 \\
\hline SNRPD1 & High vs. low & $1.897(1.130-3.182)$ & 0.015 & $1.642(1.049-2.571)$ & 0.030 \\
\hline \multicolumn{6}{|l|}{ Multivariate analysis } \\
\hline TNM staging & I/II vs. III & $1.997(1.136-3.511)$ & 0.016 & $1.682(1.049-2.699)$ & 0.031 \\
\hline Tumor differentiation & Hihg vs. median/low & $1.070(0.519-2.206)$ & 0.854 & $1.166(0.624-2.180)$ & 0.631 \\
\hline Vascular invasion & Yes vs. no & $1.257(0.713-2.216)$ & 0.430 & $1.861(1.122-3.087)$ & 0.016 \\
\hline Tumor encapsulation & Yes vs. no & & & $0.209(0.129-0.338)$ & $<0.001$ \\
\hline SNRPD1 & High vs. low & $1.890(1.098-3.255)$ & 0.022 & $1.735(1.070-2.813)$ & 0.026 \\
\hline
\end{tabular}

Abbreviations: HR, hazard ratio; CI, confidential interval; AFP - alpha fetoprotein, TNM - tumor, node, metastasis. ${ }^{*} P$-Value $<0.05$ were considered statistically significant. 
Table 5. The main GO and KEGG pathway enrichment analysis for 81 co-expressed genes.

\begin{tabular}{|c|c|c|c|c|c|c|}
\hline Category & ID Term & Term & Count & ${ }^{*} P$-Value & Benjamini & FDR \\
\hline GOTERM_Biological Process & GO:0051301 & Cell division & 21 & 6.60E-17 & $3.40 \mathrm{E}-14$ & 3.25E-14 \\
\hline GOTERM_Biological Process & GO:0051170 & Nuclear import & 8 & $9.85 \mathrm{E}-13$ & $2.54 \mathrm{E}-10$ & $2.43 \mathrm{E}-10$ \\
\hline GOTERM_Biological Process & GO:0000398 & mRNA splicing, via spliceosome & 14 & 2.31E-11 & 3.97E-09 & $3.80 \mathrm{E}-09$ \\
\hline GOTERM_Biological Process & GO:0007067 & Mitotic nuclear division & 13 & 1.30E-09 & $1.67 \mathrm{E}-07$ & $1.60 \mathrm{E}-07$ \\
\hline GOTERM_Biological Process & GO:0000387 & Spliceosomal snRNP assembly & 7 & 2.65E-09 & 2.74E-07 & 2.61E-07 \\
\hline GOTERM_Biological Process & GO:0006364 & rRNA processing & 9 & $6.04 \mathrm{E}-06$ & $4.45 \mathrm{E}-04$ & $4.25 \mathrm{E}-04$ \\
\hline GOTERM_Biological Process & GO:0008334 & Histone mRNA metabolic process & 4 & $1.98 \mathrm{E}-05$ & $1.28 \mathrm{E}-03$ & $1.22 \mathrm{E}-03$ \\
\hline GOTERM_Biological Process & GO:0006369 & Termination of RNA polymerase II transcription & 5 & 2.11E-04 & 7.69E-03 & 7.34E-03 \\
\hline GOTERM_Biological Process & GO:0000245 & Spliceosomal complex assembly & 4 & 2.23E-04 & 7.69E-03 & 7.34E-03 \\
\hline GOTERM_Biological Process & GO:0008283 & Cell proliferation & 9 & $2.68 \mathrm{E}-04$ & $8.64 \mathrm{E}-03$ & $8.25 \mathrm{E}-03$ \\
\hline GOTERM_Biological Process & GO:0008380 & RNA splicing & 6 & 9.89E-04 & $2.55 \mathrm{E}-02$ & $2.44 \mathrm{E}-02$ \\
\hline GOTERM_Biological Process & GO:0006281 & DNA repair & 6 & 4.49E-03 & $9.26 \mathrm{E}-02$ & $8.85 \mathrm{E}-02$ \\
\hline GOTERM_Biological Process & GO:0051726 & Regulation of cell cycle & 4 & 1.93E-02 & 2.77E-01 & 2.65E-01 \\
\hline KEGG_PATHWAY & hsa03040 & Spliceosome & 12 & 2.87E-09 & $1.95 \mathrm{E}-07$ & $1.95 \mathrm{E}-07$ \\
\hline KEGG_PATHWAY & hsa03010 & Ribosome & 6 & $3.46 \mathrm{E}-03$ & $1.18 \mathrm{E}-01$ & $1.18 \mathrm{E}-01$ \\
\hline KEGG_PATHWAY & hsa04110 & Cell cycle & 5 & 1.40E-02 & 3.17E-01 & 3.17E-01 \\
\hline
\end{tabular}

${ }^{*} P$-Value $<0.05$ were considered statistically significant.

A

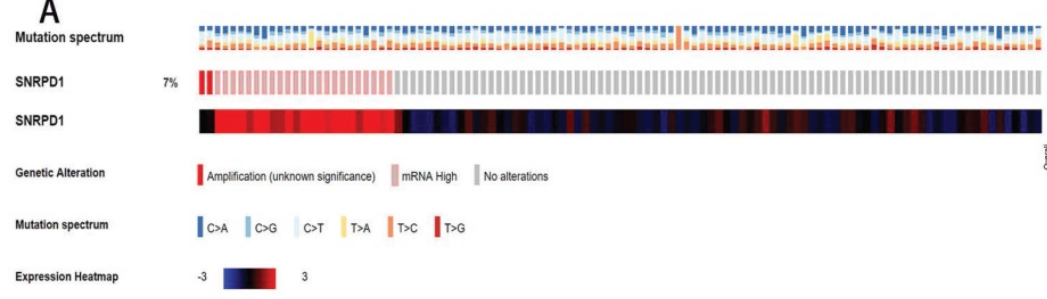

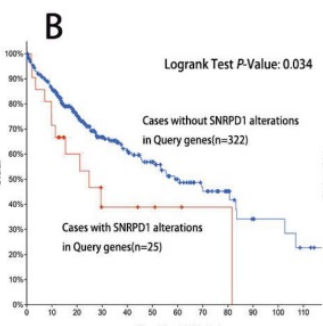

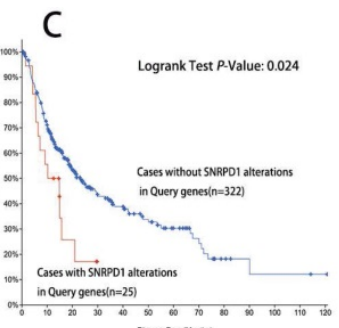

E

F
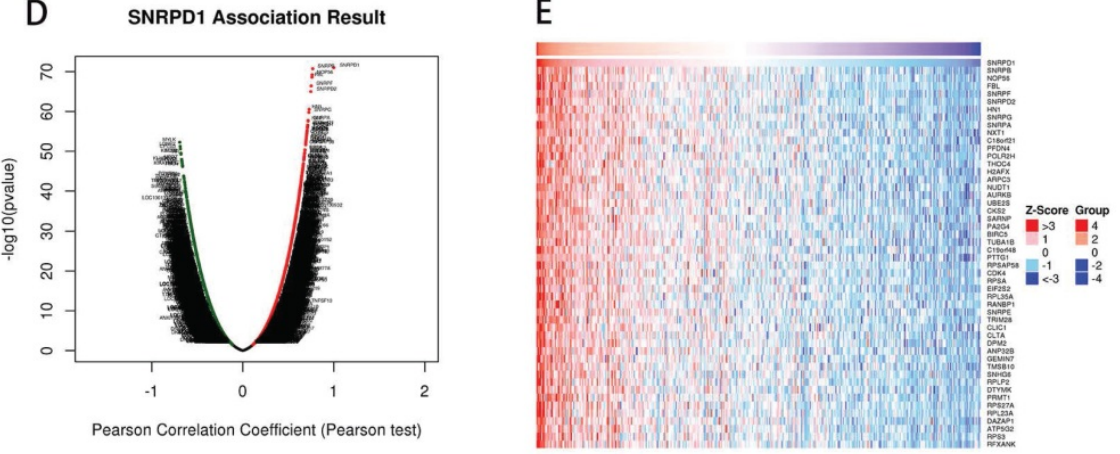

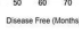

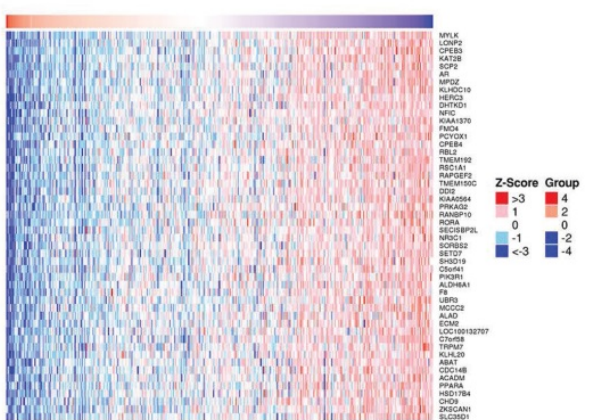

G

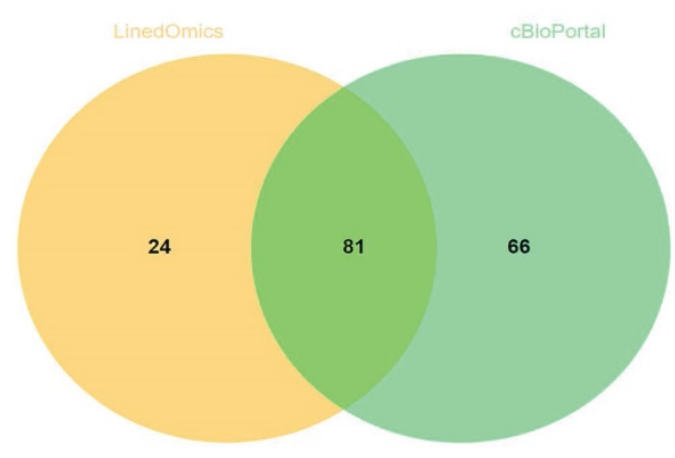

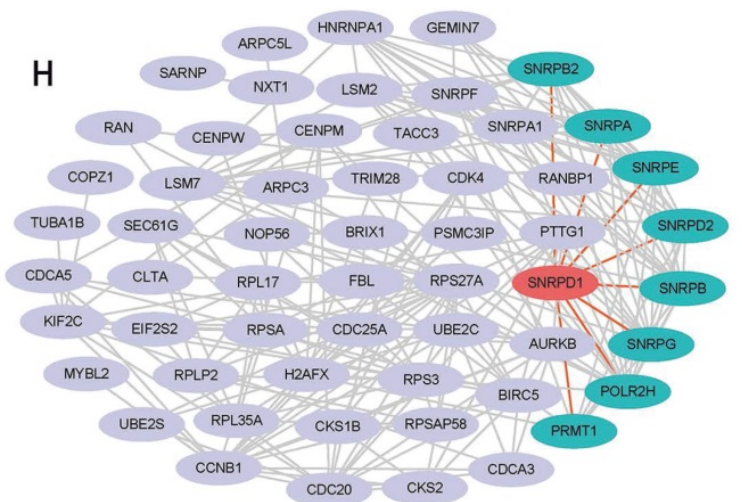

Figure 3. Alteration analyzed of SNRPDI and identification of co-expressed genes and construction of protein-protein interaction (PPI) network. (A) The mutation spectrum, alteration and expression heatmap of SNRPD1 in a cohort of 349 HCC patients in the cBioPortal database. $7 \%$ cases of this cohort exhibited alteration, including 2 cases of amplification, and 23 cases of mRNA high expression. (B-C) The overall survival (B), and the disease-free survival (C) of HCC patients with SNRPDI alterations ( $=25)$, was poorer than without SNRPDI alterations $(n=324)$. (D-F) SNRPDI expression associated target genes analysis in the LinkedOmics database. (D) Volcano chart exhibited SNRPDI expression positively/negatively correlated significant genes. (E) The top 50 genes that are positively associated with SNRPDI expression. (F) The top 50 genes that are negatively associated with SNRPDI expression. (G)81 overlapping correlated genes with Spearman's Correlation greater than 0.6 were screened as SNRPDI co-expressed genes. (H) PPI network for 81 co-expressed genes of SNRPD1 was constructed and visualized. SNRPA, SNRPB, SNRPB2, SNRPD2, SNRPE, SNRPG, POLR2H, and PRMT1 protein can interact with SNRPD1. 

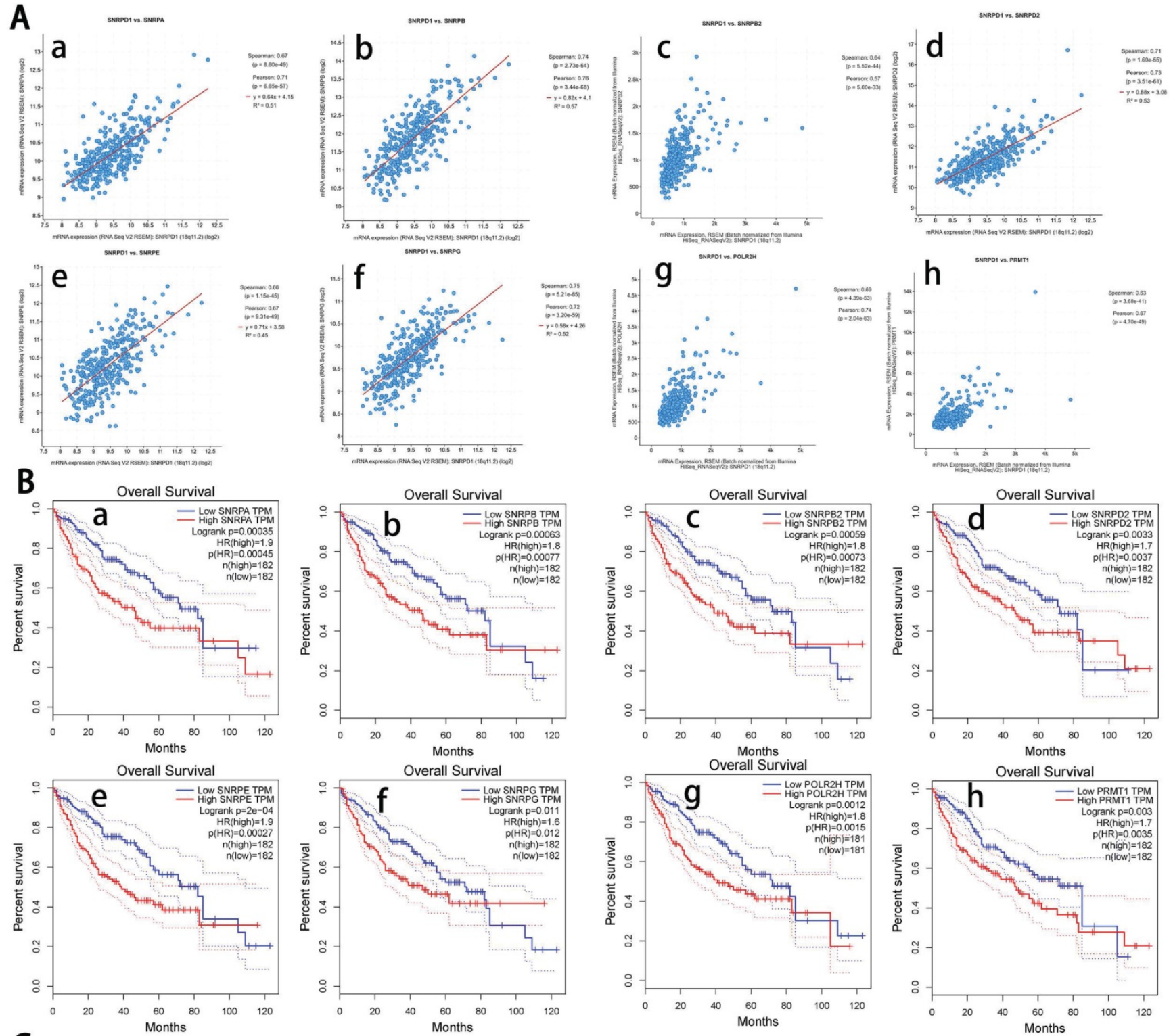

C
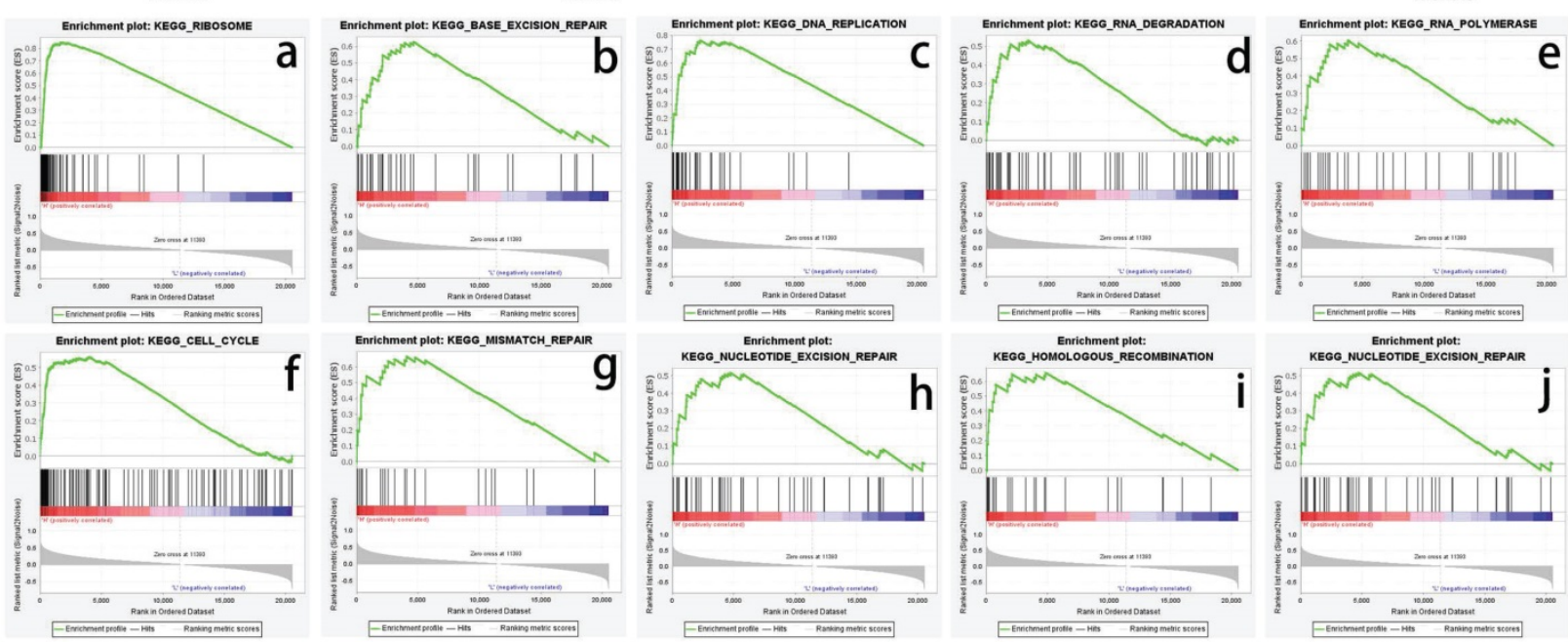

Figure 4. Survival analysis of co-expressed genes interacted with SNRPDl and KEGG pathways analysis using GSEA. (A) Correlation between SNRPDI and SNRPA (a, $r=0.71$ ), SNRPB (b, $r=0.76$ ), SNRPB2 (c, $r=0.64)$, SNRPD2 ( $d, r=0.73$ ), SNRPE (e, $r=0.67)$, SNRPG (f, $r=0.75)$, POLR2H (g, $r=0.74)$, and PRMT1 (h, $r=0.67)$, all P<0.05. (B) The survival analysis showed that the mRNA expression of SNRPA (a), SNRPB (b), SNRPB2 (c), SNRPD2 (d), SNRPE (e), SNRPG (f), POLR2H (g), and PRMT1 (h) were significantly related to the OS in HCC patients (all P<0.05). (C) The main enriched KEGG pathways of SNRPDI using GSEA. RIBOSOME (a), BASE EXCISION REPAIR (b), DNA_REPLICATION (c), DNA REPLICATION (d), RNA POLYMERASE (e), CELL CYCLE (f), MISMATCH REPAIR (g), NUCLEOTIDE EXCISION REPAIR (h), HOMOLOGOUS RECOMBINATION (i), BLADDER CANCER (j). 
A

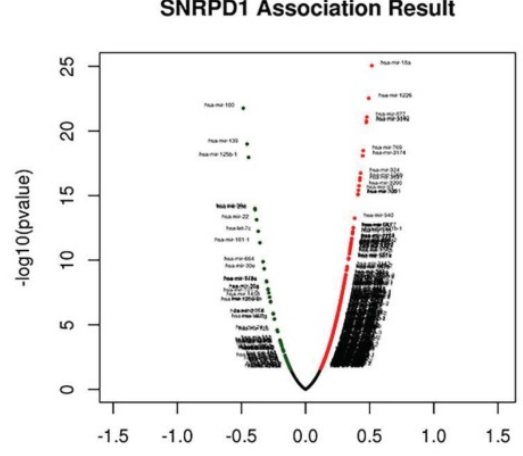

D

Pearson Correlation Coefficient (Pearson test)

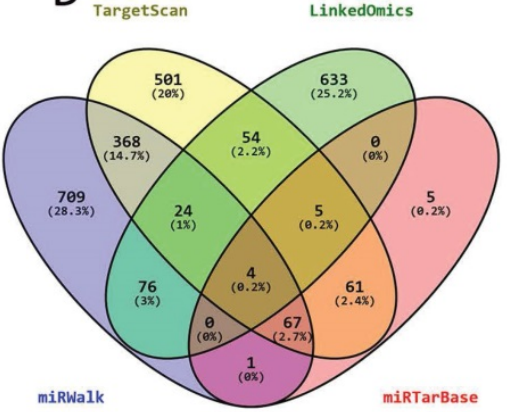

B

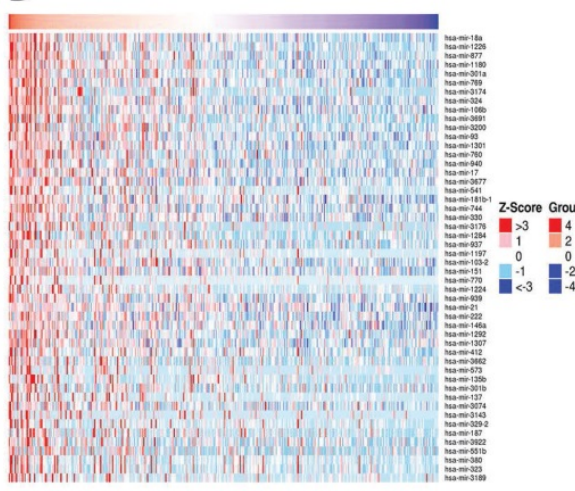

$\mathrm{E}$

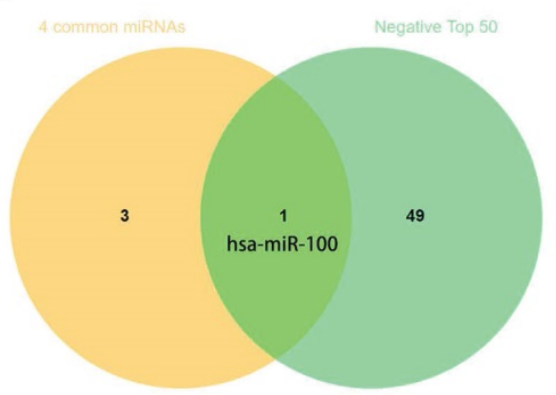

C

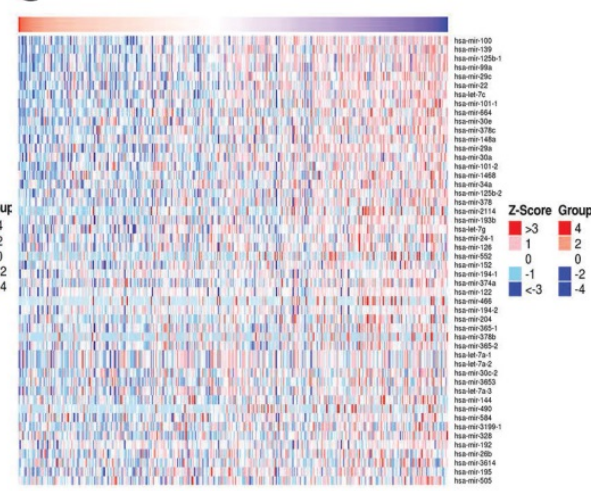

$\mathrm{F}$
G ROC curve: TCGA database

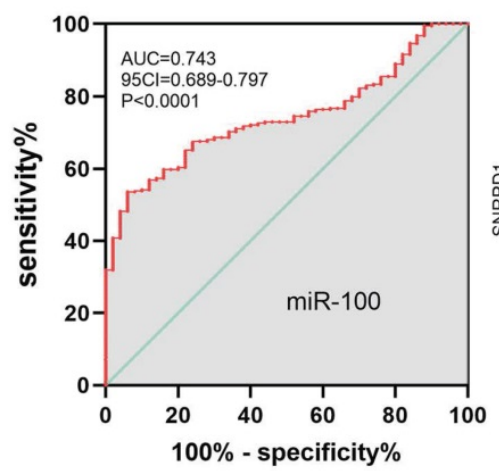

」 hsa-mir-100 vs overall_survival

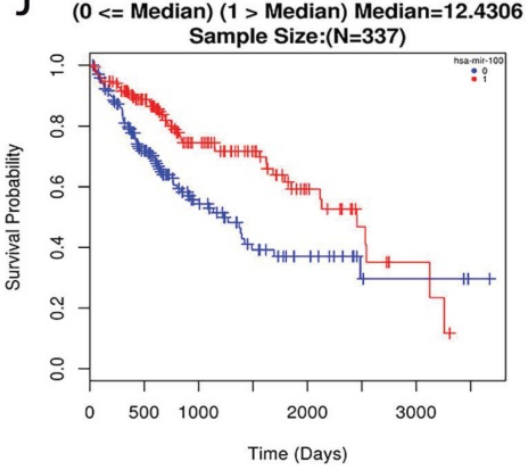

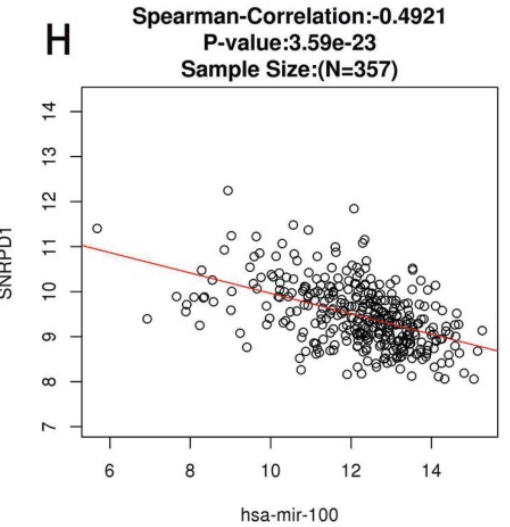

K hsa-mir-100 vs pathology_T_stage Sample Size:( $\mathrm{N}=35 \overline{9})$

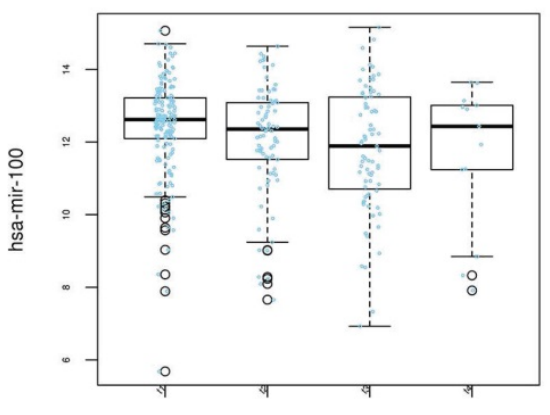

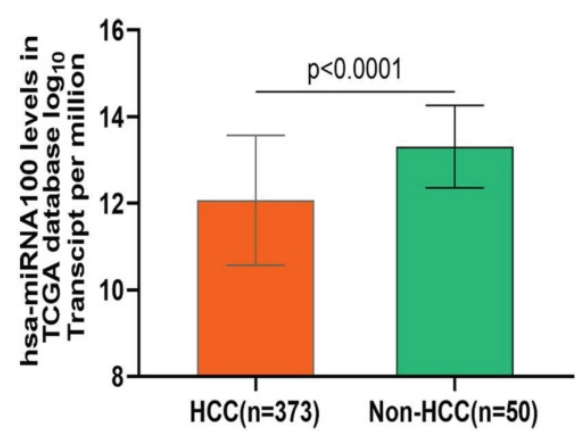

Q Statistics $\quad{ }^{*} P$-Value $\quad F D R(B H)$ \begin{tabular}{lllll}
\hline overall_survival (Cox Regression Test) & $-1.767 \mathrm{e}-01$ & $8.925 \mathrm{e}-04$ & $9.818 \mathrm{e}-03$
\end{tabular} pathology_T_stage (Kruskal-Wallis Test) $\quad 1.010 \mathrm{e}+01 \quad 1.774 \mathrm{e}-02 \quad 6.515 \mathrm{e}-02$ pathologic_stage (Kruskal-Wallis Test) $\quad 1.010 \mathrm{e}+01 \quad 1.777 \mathrm{e}-02 \quad 6.515 \mathrm{e}-02$ pathology_N_stage (Wilcox Test) $\quad-1.807 \mathrm{e}-01 \quad 3.384 \mathrm{e}-02 \quad 9.307 \mathrm{e}-02$ years_to_birth (Spearman Correlation) $\quad 8.605 \mathrm{e}-02 \quad 1.165 \mathrm{e}-01 \quad 2.563 \mathrm{e}-01$ $\begin{array}{llll}\text { race (Kruskal-Wallis Test) } & 5.088 \mathrm{e}+00 & 1.655 \mathrm{e}-01 & 3.033 \mathrm{e}-01\end{array}$ Tumor_purity (Spearman Correlation) $\quad 4.436 \mathrm{e}-02 \quad 4.190 \mathrm{e}-01 \quad 6.585 \mathrm{e}-01$ residual_tumor (Kruskal-Wallis Test) $\quad 1.034 \mathrm{e}+00 \quad 5.962 \mathrm{e}-01 \quad 7.299 \mathrm{e}-01$ radiation_therapy (Wilcox Test) $\quad-3.172 \mathrm{e}-02 \quad 6.410 \mathrm{e}-01 \quad 7.299 \mathrm{e}-01$ pathology_M_stage (Wilcox Test) $\quad-4.300 \mathrm{e}-03 \quad 6.636 \mathrm{e}-01 \quad 7.299 \mathrm{e}-01$ FDR(BH)-False Discovery Rate is calculated by BH(Benjamini-hochberg method).

hsa-mir-100 vs pathologic_stage Sample Size:( $\mathrm{N}=338)$

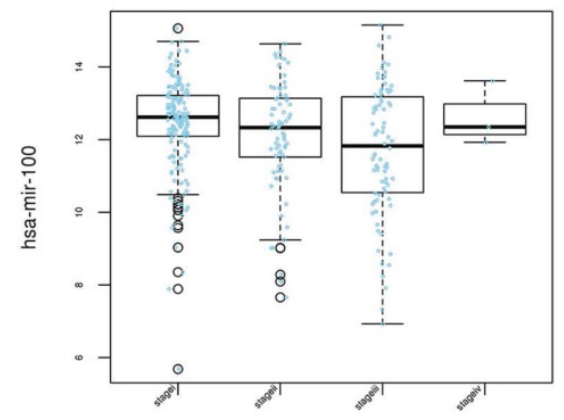

Figure 5. Analysis of association between miRNAs and SNRPDI expression in the TCGA database. (A-C) SNRPDI expression associated miRNAs analysis in the LinkedOmics database. (A) Volcano chart exhibited SNRPDI expression positively/negatively correlated significant miRNAs. (B) The top 50 miRNAs that are positively associated with SNRPD1 expression. (C) The top 50 miRNAs that are negatively associated with SNRPD1 expression. (D) 4 overlapping miRNAs interact with SNRPD1 obtained from the "LinkedOmics", "miRWalk", "TargetScan", and "miRTarBase" databases. (E) The Venny diagram exhibited that hsa-miR-100 overlapping in "4 Common miRNAs" and "SNRPD1 Negatively Correlated Significant miRNAs (top 50)". (F) The expression of miR-100 in HCC tissues ( $n=373$ ) was significantly lower than that in non-HCC tissues ( $=50$ ) in the TCGA database. $(G)$ The receiver operating characteristic $(R O C)$ curve revealed that miR-100 expression has a significant diagnosis value on $\mathrm{HCC}(\mathrm{AUC}=0.743$, $\mathrm{P}<0.0001)$. $(\mathrm{H})$ Scatter plot visualizing that hsa-mir-100 negatively significantly correlated with SNRPDI expression in the HCC patients (Spearman-correlation: -0.4921 , P=3.59e-23). (I) Association of miR-100 expression with clinicopathologic outcomes in HCC patients in the LinkedOmics database. (J) The survival analysis showed that low hsa-miR-100 mRNA expression were significantly associated with the poor overall survival in $\mathrm{HCC}$ patients $(\mathrm{P}=8.925 \mathrm{e}-04)$. $(\mathrm{K}) \mathrm{H}$ sa-miR-100 expression was correlated with pathology_T_stage $(\mathrm{P}=$ 1.774E-02). (L) Hsa-miR-100 expression was correlated with pathologic_stage $(P=1.777 \mathrm{E}-02)$. 

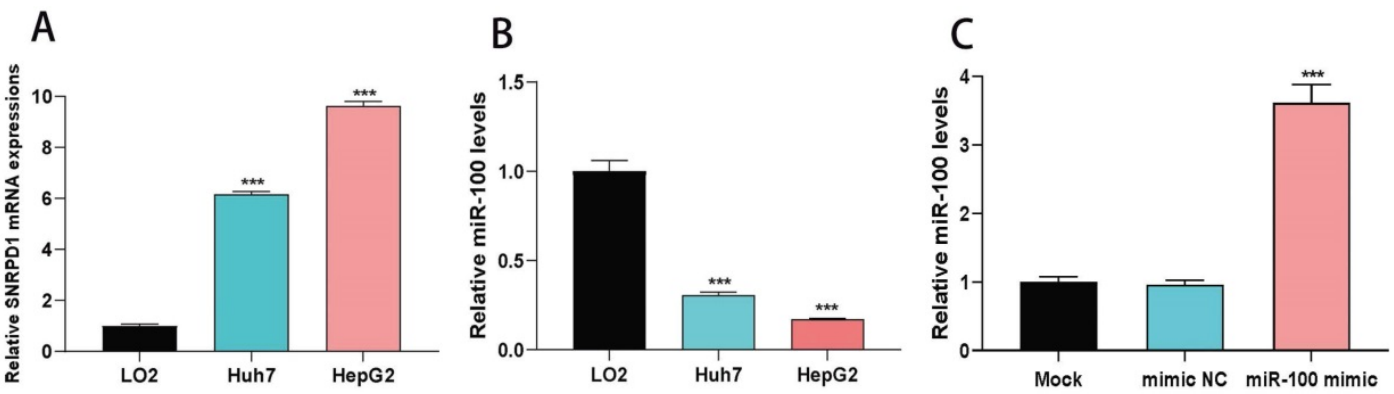

D

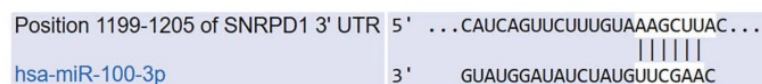

$\mathrm{E}$

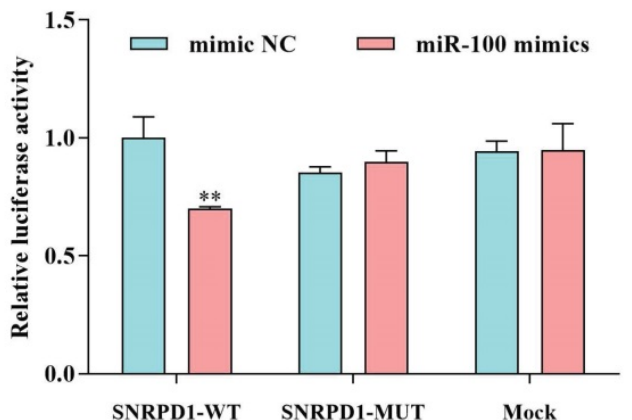

$\mathrm{F}$

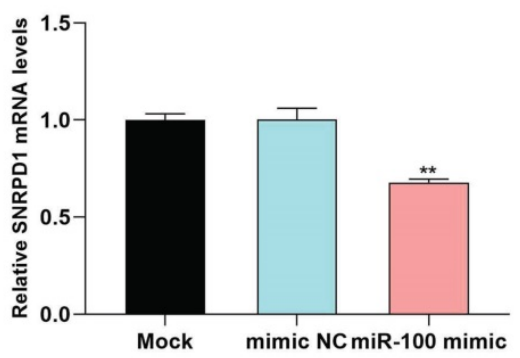

Figure 6. Correlations between miR-100 and SNRPDI expression in HCC cells. (A) mRNA expression of SNRPD1 in HCC cells was significantly higher than normal liver cells $(\mathrm{P}<0.001)$. (B) mRNA expression of miR-100 in HCC cells was significantly lower than normal liver cells $(\mathrm{P}<0.001)$. (C) Expression of miR-100 was significantly upregulated by cell transfection with miR-100 mimic $(P<0.001)$. (D) Predicted complementary sequences of hsa-miR-100-3p in 3'-UTR of SNRPD1. (E) The HepG2 cells transfected by miR-100 mimic were significantly decreased luciferase activity of WT 3'-UTR of SNRPD1 (P<0.01). (F) The upregulation of miR-100 expression in HepG2 cells by transfected miR-100 mimics resulted in significantly decreased expression of SNRPD1 $(\mathrm{P}<0.01)$.

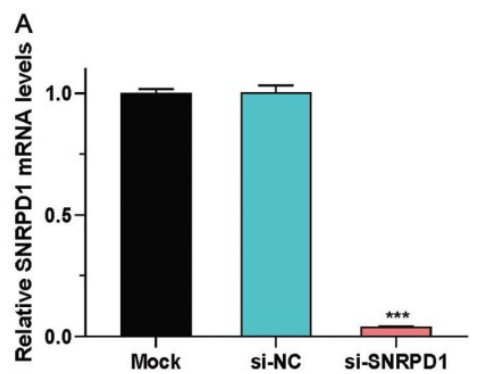

B

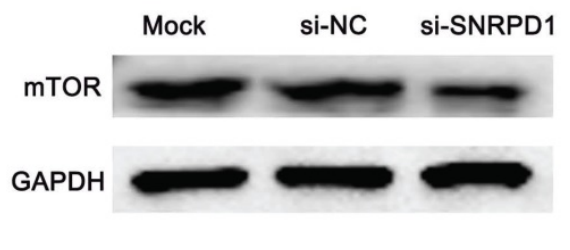

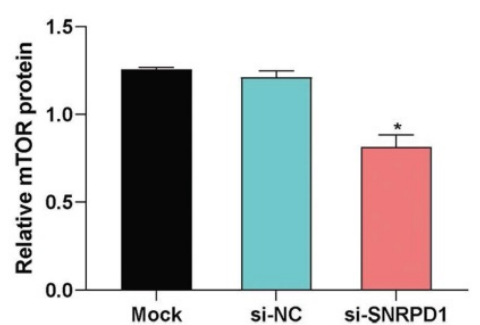

Figure 7. Effect of SNRPDI on mTOR signaling pathway. (A) The siRNA of SNRPD1 (si-SNRPDI) successfully inhibited mRNA expression of SNRPD1 in HepG2 cells. (B) The western blot analysis exhibited the mTOR protein expression in HepG2 cells. (C) si-SNRPD1 significantly decreased the mTOR protein expression in HepG2 cells.

Table 6. The main enriched KEGG pathways of SNRPDI High Expression in TCGA database.

\begin{tabular}{lllll}
\hline KEGG enrichment signal pathway & ES & NES & NOM p-value & FDR q-value \\
\hline KEGG_RIBOSOME & 0.848 & 1.788 & 0.016 & 0.086 \\
KEGG_BASE_EXCISION_REPAIR & 0.627 & 1.857 & 0.004 & 0.083 \\
KEGG_DNA_REPLICATION & 0.763 & 1.839 & 0.008 & 0.077 \\
KEGG_RNA_DEGRADATION & 0.532 & 1.817 & 0.004 & 0.076 \\
KEGG_RNA_POLYMERASE & 0.603 & 1.785 & 0.008 & 0.067 \\
KEGG_CELL_CYCLE & 0.560 & 1.775 & 0.014 & 0.066 \\
KEGG_MISMATCH_REPAIR & 0.665 & 1.718 & 0.028 & 0.095 \\
KEGG_NUCLEOTIDE_EXCISION_ & 0.516 & 1.607 & 0.037 & 0.193 \\
REPAIR & & & & \\
KEGG_HOMOLOGOUS_ & 0.658 & 1.729 & 0.019 & 0.095 \\
RECOMBINATION & & & & \\
KEGG_BLADDER_CANCER & 0.496 & 1.639 & 0.010 & 0.164
\end{tabular}

${ }^{*} P$-Value $<0.05$ were considered statistically significant.

\subsection{SNRPDI knockdown promotes autophagy of HCC cells.}

We used transmission electron microscopy (TEM) to detect the ultrastructural details of autophagosomes to evaluate the influence of SNRPD1 knockdown on autophagy. As shown in Figure. 8A, HepG2 cells transfected with si-SNRPD1 exhibited a remarkable increase number of autophagosomes and autolysosomes, compared with si-NC cells (Fig. 8A). At the same time, the rates of autophagosomes found in cells transfected with si-SNRPD1 and si-NC were $11 \%$ and $4 \%$, respectively. We next analyzed the effect of si-SNRPD1 transfection on the expression of Microtubule-Associated Protein 1 Light Chain 3 (LC3), commonly used as a critical protein involved in 
the process of autophagy and directly participated in the formation of autophagosomes [34, 35]. Consistent with the results of TEM, western blotting analyses showed that si-SNRPD1 transfected in HepG2 cells significantly decreased SNRPD1 protein level, but significantly increased expression of LC3-I, LC3-II protein, and LC3-II/LC3-I ratio (Fig. 8B-D). The results of real-time PCR analysis implicated that Atg 5, Atg 7, and Atg 12 mRNA levels significantly elevated in si-SNRPD1 transfected cells (Fig. 8E). Overall, these data indicated that SNRPD1 knockdown might promote autophagy of HCC cells.
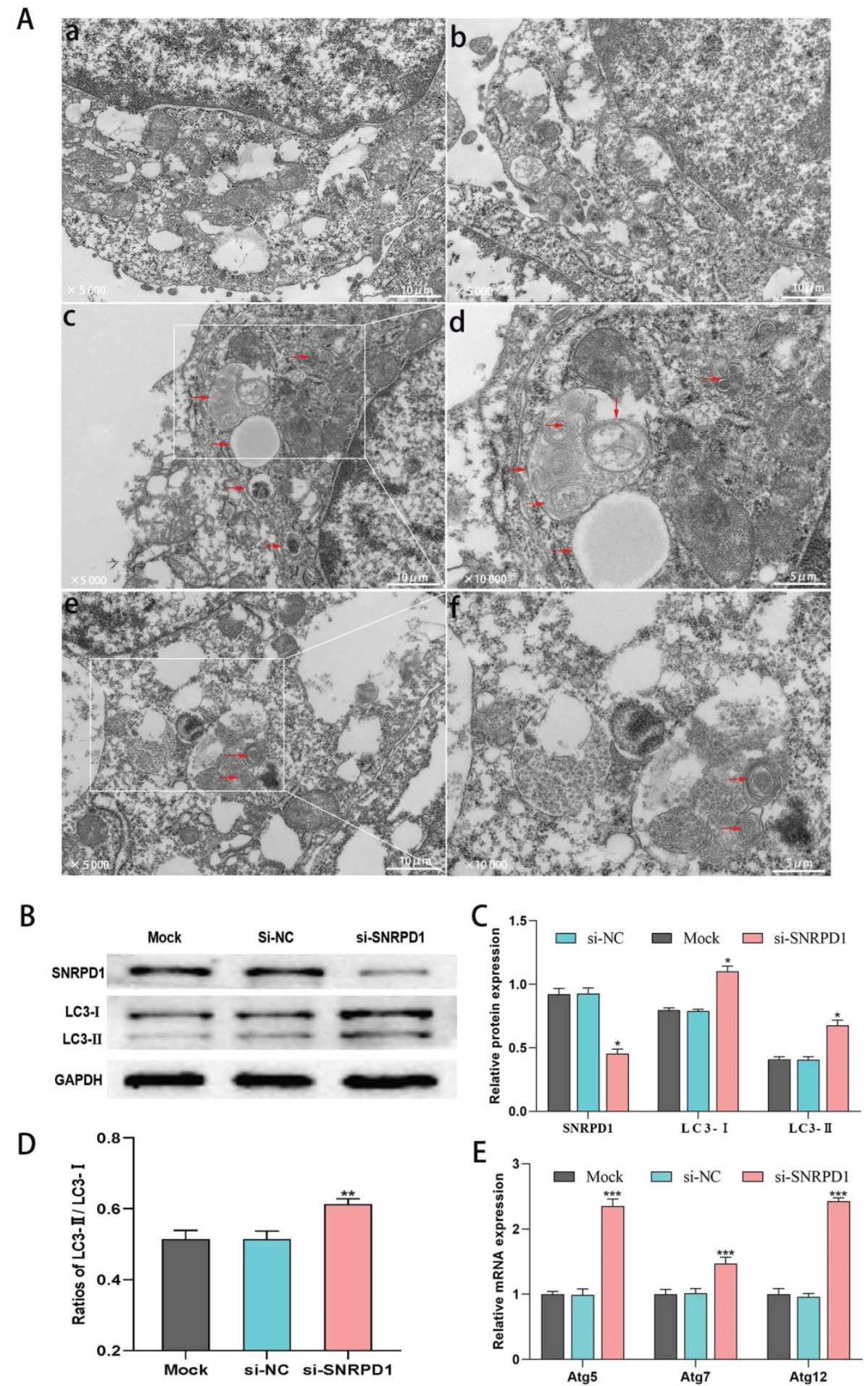

Figure 8. siRNA of SNRPD1 (si-SNRPD1) promotes autophagy in HepG2 cells. (A) Transmission electron microscopy (TEM) was used to detect the autophagic events characterized. HepG2 cells were transfected with si-SNRPD1 or without si-SNRPD1 (si-NC). Compared with si-NC HepG2 cells (a, b), autophagosomes and autolysosomes were mainly observed in si-SNRPD1 cells (c, $d, e, f$ ). HepG2 cells transfected by si-SNRPD1 or si-NC were shown at 2 magnifications, $x 5,000$ (a, b, c, e) and $x 10,000$ ( $d, f$ ). (B) The western blot analysis exhibited the LC3-I and LC3-II protein expression in HepG2 cells transfected by si-SNRPD1 or si-NC. (C-D) LC3-I, LC3-II protein expression (C, $\mathrm{P}<0.05)$, and LC3-II/LC3-I ratio $(\mathrm{D}, \mathrm{P}<0.01)$ significantly increased in si-SNRPD1 transfected cells. (E) The real-time PCR analysis implicated that Atg 5, Atg 7, and Atg 12 mRNA levels significantly elevated in si-SNRPD1 transfected cells (all $\mathrm{P}<0.001$ ). 


\section{Discussion}

Spliceosome was responsible for participating in the splicing of the pre-messenger RNA, such as removing introns and then the connection of exons in a certain order [3-5]. The process of RNA splicing governs many aspects of cellular proliferation, survival, and differentiation. Alteration of this process has been proved implicating in many human cancers [36-38]. SNRPB and SNRPE, both the core components of the spliceosome, were significantly overexpressed in HCC tissues and associated with a worse prognosis $[13,14]$. SNRPD1 also was a crucial gene that regulates the assembly of the pluripotency-specific spliceosome and acquires and maintains pluripotency [19]. Ming Yi and colleagues constructed a gene co-expression network using weighted gene co-expression Network Analysis showed that SNRPD1 was a predictive biomarker of lung adenocarcinoma due to its high expression was associated with poor prognosis [39]. Studies have shown that SNRPD1 overexpression promotes the development of breast cancer by cooperating with genes involved in the cell cycle, mitosis, and chromatin replication. And silencing SNRPD1 in breast cancer cells may cause tumor cell growth to stop and cell cycle arrest in the G0/G1 stage [40]. However, no previous study has addressed the role of SNRPD1 in HCC and its prognostic and diagnostic value. This study is the first systematic investigation of the associations between SNRPD1 expression in mRNA and protein level with clinical outcomes, diagnostic and prognostic value in HCC patients.

SNRPD1 mRNA expression in HCC was significantly higher than in normal liver tissues. Otherwise, its expression is incrementally upregulated with increasing tumor stages. Furthermore, the investigation of protein expression of SNRPD1 in the human protein atlas database showed that its expression higher than normal liver tissues, which was validated by our IHC of 154 HCC patients. Higher mRNA and protein expression of SNRPD1 were both associated with poor OS and RFS of HCC patients. The ROC curve revealed that SNRPD1 mRNA expression has a significant diagnostic value on HCC.

High SNRPD1 mRNA expression was significantly positively correlated to the TNM stage, serum AFP level, CLIP staging. The multivariate regression analysis confirmed that the high SNRPD1 mRNA expression was an independent risk factor for OS and RFS in HCC patients. The analysis of clinicopathologic characteristics in 154 HCC patients revealed that high SNRPD1 protein expression was significantly correlated to TNM staging, serum AFP level, tumor differentiation, vascular invasion, recurrence, and survival. IHC was routine pathological examination after liver cancer resection. Our result demonstrated that high SNRPD1 protein expression was an independent risk factor for OS and RFS of HCC patients. Therefore, postoperative SNRPD1 IHC examination may help to predict the prognosis and recurrence of patients with HCC.

Mutations in genes encoding the components of the splicing machinery were reported in carcinoma from various origins, such as hematologic malignancies, breast, and colon [41, 42]. Volker and colleagues showed that mutations in SNRPD1 lead to a significant dominance of $\mathrm{T}$ cells targeting the mutant epitope in melanoma patients, leading to the development of anti-tumor immunity [43]. We queried the SNRPD1 alterations in a cohort of 347 HCC patients and found that $25(7 \%)$ of queried HCC patients has the alterations. Notably, the OS and disease-free survival of HCC patients with SNRPD1 alterations were significantly lower than those without the alterations. Therefore, exploring the cause of SNRPD1 alterations may promote finding a new method for the treatment of HCC by targeting the expression and alteration of SNRPD1.

To investigate the role of SNRPD1 in the occurrence and development of HCC, GO and KEGG enrichment analysis on co-expressed genes of SNRPD1 was performed. GO analysis showed that the biological processes of SNRPD1 implicated included cell division, mRNA splicing via spliceosome, mitotic nuclear division, etc. We noticed that the KEGG pathways of DAVID and GESA indicated that SNRPD1 was involved in regulating the cell cycle, consistent with the previous study. SNRPD1 promotes the progression of breast cancer by regulating the cell cycle [40]. Based on the above research results, it is reasonable to hypothesize that the overexpression of SNRPD1 may lead to the overexpression of genes that promote the cell cycle by causing changes in RNA splicing and promote the progression of HCC, which was worth further study.

MicroRNAs (miRNAs), highly conserved small non-protein-coding RNAs, play a crucial role in negatively regulating various cancers through targeting a wide range of target genes [44, 45]. Therefore, the identification of miRNAs that regulating the occurrence and development of HCC may provide new methods for the diagnosis and treatment of HCC. Ke R and colleagues found that miR-22 was significantly down-regulated and promoted the progress of HCC by negatively targeting the expression of HNRNPA1 [46]. Previous studies have found that miR-100 plays a role in suppressor in HCC $[47,48]$. Our research-based on 
bioinformatics analysis found that miR-100 directly targets and negatively regulates the expression of SNRPD1 in HCC. The miR-100 expression was significantly down-regulated in HCC. The ROC curve shows that miR-100 has a significant diagnostic value for HCC. Furthermore, low miR-100 expression was associated with poor overall survival, pathologic stage, pathology $\mathrm{T}$ stage, and pathology $\mathrm{N}$ stage in HCC. Our luciferase reporter assay revealed that SNRPD1 was the direct target gene of miR-100 in HCC cells. This result was consistent with bioinformatics.

Previous studies found that SNRPD1 knockdown can inhibit the mTOR pathway and promote autophagy in breast cancer SKBr-3 cell lines [3]. Previous studies have reported that miR-100 regulate the mTOR signaling pathway in a variety of tumor types. Ye et al. reported that miR-100 downregulates mTOR to suppress the proliferation, migration, and Invasion of prostate cancer Cells [49]. $\mathrm{Yu}$ et al. demonstrated that miR-100 up-regulation enhanced cell autophagy and apoptosis induced by cisplatin in osteosarcoma by targeting mTOR [50]. In addition, Lin et al. reported that miR-100 inhibits cell proliferation in mantle cell lymphoma by targeting mTOR [51]. Another study revealed that miR-100 promotes the autophagy of hepatocellular carcinoma cells by inhibiting the expression of the mTOR pathway [52]. Therefore, we supposed that SNRPD1 acts as an oncogene in the occurrence and progression of HCC through regulates the mTOR pathway and autophagy. Our western blot analysis showed that mTOR was significantly downregulated after SNRPD1 was knockdown, whereas LC3-I, LC3-II was significantly upregulated, and LC3-II/LC3-I ratio was increased. Furthermore, HCC cells transfected with si-SNRPD1 had a remarkable increase number of autophagosomes and autolysosomes, compared with si-NC cells, which was confirmed by TEM. Overall, all our results indicating that SNRPD1 acts as an oncogene in the occurrence and progression of HCC through regulates the mTOR pathway and autophagy.

\section{Conclusion}

In summary, our study demonstrates that SNRPD1 expression was significantly upregulated in HCC compared with adjacent normal liver tissues. High SNRPD1 expression was associated with poor clinical outcomes. SNRPD1 may regulate the progression of HCC by influencing the process of cell cycle and mRNA splicing via spliceosome, etc. SNRPD1 was the direct target gene of miR-100 in HCC cells. SNRPD1 may as an oncogene regulating the tumorigenesis and progression of HCC through regulates the mTOR pathway and autophagy, suggesting that designing new drugs targeting SNRPD1 may provide new insight and methods for the treatment of HCC.

\section{Abbreviations}

SNRPD1: Small nuclear ribonucleoprotein Sm D1; HCC: hepatocellular carcinoma; IHC: immunohistochemistry; GSEA: Gene set enrichment analysis; HBV: hepatitis B virus; $\mathrm{HCV}$ : hepatitis C virus; AFP: Serum alpha-fetoprotein; GEO: Gene Expression Omnibus; GO: gene ontology; KEGG: The Kyoto Encyclopedia of Genes and Genomes; GEPIA: Gene Expression Profiling Interactive Analysis; TCGA: The Cancer Genome Atlas; CCLE: The Cancer Cell Line Encyclopedia project; OS: overall survival; RFS: recurrence-free survival; DAVID: Database for Annotation, Visualization and Integrated Discovery; PPI: protein-protein interaction; qRT-PCR: Quantitative polymerase chain reaction; TEM: transmission electron microscopy; LC3: Protein 1 Light Chain 3.

\section{Acknowledgements}

The authors are grateful to the contributors of the GSE14520 and TCGA for sharing the HCC expression profile data set on open access. In addition, we would like to acknowledge to all the people who have given us help on our article.

\section{Availability of data and materials}

All data generated or analyzed during this study are included in this published article.

\section{Ethics approval and consent to participate}

This study was performed according to the relevant medical ethics regulations and approved by the Human Research Ethics Committee of 900 Hospital of the Joint Logistics Team (Fuzhou, China). All participants gave written informed consent prior to surgery and collection of the specimens.

\section{Funding}

This work was supported by The Key Project of Natural Science Foundation of Fujian Province, China (no. 2020Y0078 and 2020J011144).

\section{Author Contributions}

Conception and design: Yi Jiang, Huanzhnag $\mathrm{Hu}$; administrative support: $\mathrm{Yi}$ Jiang; Huanzhnag $\mathrm{Hu}$; provision of study materials or patients: Huaxiang Wang, Fengfeng $\mathrm{Xu}$; collection and assembly of data: Huaxiang Wang, Fengfeng $\mathrm{Xu}$, Lingling Lu; data analysis and interpretation: Huaxiang Wang, Fengfeng $\mathrm{Xu}$, Lingling Lu, Fang Yang; supplementary mechanism experiment: Huaxiang Wang, Fengfeng 
Xu, Xinghua Huang; Lizhi Lv; manuscript writing: Huaxiang Wang, Fengfeng $\mathrm{Xu}$, Lingling Lu; writing review \& editing: Yi Jiang, Huanzhnag $\mathrm{Hu}$; final approval of manuscript: All authors.

\section{Competing Interests}

The authors have declared that no competing interest exists.

\section{References}

1. Yang JD, Hainaut P, Gores GJ, Amadou A, Plymoth A, Roberts LR. A global view of hepatocellular carcinoma: trends, risk, prevention and management. Nat Rev Gastroenterol Hepatol. 2019; 16: 589-604.

2. Kulik L, El-Serag HB. Epidemiology and Management of Hepatocellular Carcinoma. Gastroenterology. 2019; 156: 477-91.

3. Ouidville V, Alsafadi S, Goubar A, Commo F, Scott V, Pioche-Durieu C, et al. Targeting the deregulated spliceosome core machinery in cancer cells triggers mTOR blockade and autophagy. Cancer Res. 2013; 73: 2247-58

4. Will CL, Luhrmann R. Spliceosome structure and function. Cold Spring Harb Perspect Biol. 2011; 3: 1-23.

5. Wan R, Bai R, Shi Y. Molecular choreography of pre-mRNA splicing by the spliceosome. Curr Opin Struct Biol. 2019; 59: 124-33.

6. Salgado-Garrido J, Bragado-Nilsson E, Kandels-Lewis S, Seraphin B. Sm and Sm-like proteins assemble in two related complexes of deep evolutionary origin. EMBO J. 1999; 18: 3451-62.

7. Hermann H, Fabrizio P, Raker VA, Foulaki K, Hornig H, Brahms H, et al. snRNP Sm proteins share two evolutionarily conserved sequence motifs which are involved in Sm protein-protein interactions. EMBO J. 1995; 14: 2076-88.

8. Dvinge H, Guenthoer J, Porter PL, Bradley RK. RNA components of the spliceosome regulate tissue- and cancer-specific alternative splicing. Genome Res. 2019; 29: 1591-604

9. Engreitz JM, Haines JE, Perez EM, Munson G, Chen J, Kane M, et al. Local regulation of gene expression by lncRNA promoters, transcription and splicing. Nature. 2016; 539: 452-5.

10. Lee SC, Abdel-Wahab O. Therapeutic targeting of splicing in cancer. Nat Med. 2016; 22: 976-86

11. Sveen A, Kilpinen S, Ruusulehto A, Lothe RA, Skotheim RI. Aberrant RNA splicing in cancer; expression changes and driver mutations of splicing factor genes. Oncogene. 2016; 35: 2413-27.

12. Pellagatti A, Armstrong RN, Steeples V, Sharma E, Repapi E, Singh S, et al. Impact of spliceosome mutations on RNA splicing in myelodysplasia: dysregulated genes/pathways and clinical associations. Blood. 2018; 132: $1225-40$

13. Zhan YT, Li L, Zeng TT, Zhou NN, Guan XY, Li Y. SNRPB-mediated RNA splicing drives tumor cell proliferation and stemness in hepatocellular carcinoma. Aging (Albany NY). 2020; 13: 537-54

14. Jia D, Wei L, Guo W, Zha R, Bao M, Chen Z, et al. Genome-wide copy number analyses identified novel cancer genes in hepatocellular carcinoma. Hepatology. 2011; 54: 1227-36.

15. Liu N, Wu Z, Chen A, Wang Y, Cai D, Zheng J, et al. SNRPB promotes the tumorigenic potential of NSCLC in part by regulating RAB26. Cell Death Dis. 2019; $10: 667$.

16. Zhu L, Zhang X, Sun Z. SNRPB promotes cervical cancer progression through repressing p53 expression. Biomed Pharmacother. 2020; 125: 109948.

17. Li Y, Ren Z, Peng Y, Li K, Wang X, Huang G, et al. Classification of glioma based on prognostic alternative splicing. BMC Med Genomics. 2019; 12: 165

18. Hu C, Li M, Liu J, Qian J, Xu D, Zhang S, et al. Anti-SmD1 antibodies are associated with renal disorder, seizures, and pulmonary arterial hypertension in Chinese patients with active SLE. Sci Rep. 2017; 7: 7617-24.

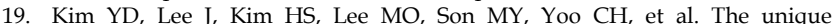
spliceosome signature of human pluripotent stem cells is mediated by SNRPA1, SNRPD1, and PNN. Stem Cell Res. 2017; 22: 43-53.

20. Batra R, Harder N, Gogolin S, Diessl N, Soons Z, Jager-Schmidt C, et al. Time-lapse imaging of neuroblastoma cells to determine cell fate upon gene knockdown. PLoS One. 2012; 7: e50988.

21. Ogata H, Goto S, Sato K, Fujibuchi W, Bono H, Kanehisa M. KEGG: Kyoto Encyclopedia of Genes and Genomes. Nucleic Acids Res. 1999; 27: 29-34

22. Tang Z, Li C, Kang B, Gao G, Li C, Zhang Z. GEPIA: a web server for cancer and normal gene expression profiling and interactive analyses. Nucleic Acids Res. 2017; 45: W98-W102.

23. Uhlen M, Fagerberg L, Hallstrom BM, Lindskog C, Oksvold P, Mardinoglu A, et al. Proteomics. Tissue-based map of the human proteome. Science. 2015; 347: 1260419.

24. Gao J, Aksoy BA, Dogrusoz U, Dresdner G, Gross B, Sumer SO, et al. Integrative analysis of complex cancer genomics and clinical profiles using the cBioPortal. Sci Signal. 2013; 6: pl1.
25. Edgar R, Domrachev M, Lash AE Gene Expression Omnibus: NCBI gene expression and hybridization array data repository. Nucleic Acids Res. 2002; 30: 207-10.

26. Ke R, Lv L, Li J, Zhang X, Yang F, Zhang K, et al. Prognostic value of heterogeneous ribonucleoprotein A1 expression and inflammatory indicators for patients with surgically resected hepatocellular carcinoma: Perspectives from a high occurrence area of hepatocellular carcinoma in China. Oncol Lett. 2018; 16: 3746-56.

27. Zhao S, Wang M, Yang Z, Tan K, Zheng D, Du X, et al. Comparison between Child-Pugh score and Albumin-Bilirubin grade in the prognosis of patients with HCC after liver resection using time-dependent ROC. Ann Transl Med. 2020; 8: 539-46.

28. Kee KM, Wang JH, Lee CM, Chen CL, Changchien CS, Hu TH, et al. Validation of clinical AJCC/UICC TNM staging system for hepatocellular carcinoma: analysis of 5,613 cases from a medical center in southern Taiwan. Int J Cancer. 2007; 120: 2650-5.

29. Vasaikar SV, Straub P, Wang J, Zhang B. LinkedOmics: analyzing multi-omics data within and across 32 cancer types. Nucleic Acids Res. 2018; 46: D956-D63.

30. Dennis G, Jr., Sherman BT, Hosack DA, Yang J, Gao W, Lane HC, et al. DAVID: Database for Annotation, Visualization, and Integrated Discovery. Genome Biol. 2003; 4: P3.

31. Franceschini A, Szklarczyk D, Frankild S, Kuhn M, Simonovic M, Roth A, et al. STRING v9.1: protein-protein interaction networks, with increased coverage and integration. Nucleic Acids Res. 2013; 41: D808-D15.

32. Goldman MJ, Craft B, Hastie M, Repecka K, McDade F, Kamath A, et al. Visualizing and interpreting cancer genomics data via the Xena platform. Nat Biotechnol. 2020; 38: 675-8.

33. Subramanian A, Tamayo P, Mootha VK, Mukherjee S, Ebert BL, Gillette MA, et al. Gene set enrichment analysis: a knowledge-based approach for interpreting genome-wide expression profiles. Proc Natl Acad Sci U S A. 2005; 102: 15545-50.

34. Barth S, Glick D, Macleod KF. Autophagy: assays and artifacts. J Pathol. 2010; 221: 117-24.

35. Jiang P, Mizushima N. LC3- and p62-based biochemical methods for the analysis of autophagy progression in mammalian cells. Methods. 2015; 75: 13-8.

36. Wang E, Aifantis I. RNA Splicing and Cancer. Trends Cancer. 2020; 6: 631-44.

37. Urbanski LM, Leclair N, Anczukow O. Alternative-splicing defects in cancer: Splicing regulators and their downstream targets, guiding the way to novel cancer therapeutics. Wiley Interdiscip Rev RNA. 2018; 9: e1476-e531.

38. Niu HT, Yang CM, Chen B, Dong Q. Biomarker research and some deduction in superficial bladder cancer cells combined with corresponding stroma. Cancer Biomark. 2011; 10: 109-16.

39. Yi M, Li T, Qin S, Yu S, Chu Q, Li A, et al. Identifying Tumorigenesis and Prognosis-Related Genes of Lung Adenocarcinoma: Based on Weighted Gene Coexpression Network Analysis. Biomed Res Int. 2020; 2020: 4169691.

40. Dai X, Yu L, Chen X, Zhang J. SNRPD1 confers diagnostic and therapeutic values on breast cancers through cell cycle regulation. Cancer Cell Int. 2021; 21: 229-44.

41. Yoshida K, Sanada M, Shiraishi Y, Nowak D, Nagata Y, Yamamoto R, et al. Frequent pathway mutations of splicing machinery in myelodysplasia. Nature. 2011; 478: 64-9.

42. Sampath J, Long PR, Shepard RL, Xia X, Devanarayan V, Sandusky GE, et al. Human SPF45, a splicing factor, has limited expression in normal tissues, is overexpressed in many tumors, and can confer a multidrug-resistant phenotype to cells. Am J Pathol. 2003; 163: 1781-90.

43. Lennerz V, Fatho M, Gentilini C, Frye RA, Lifke A, Ferel D, et al. The response of autologous $\mathrm{T}$ cells to a human melanoma is dominated by mutated neoantigens. Proc Natl Acad Sci U S A. 2005; 102: 16013-8.

44. Mishra S, Yadav T, Rani V. Exploring miRNA based approaches in cancer diagnostics and therapeutics. Crit Rev Oncol Hematol. 2016; 98: 12-23.

45. Ali Syeda Z, Langden SSS, Munkhzul C, Lee M, Song SJ. Regulatory Mechanism of MicroRNA Expression in Cancer. Int J Mol Sci. 2020; 21: 1723-40.

46. Ke RS, Zhang K, Lv LZ, Dong YP, Pan F, Yang F, et al. Prognostic value and oncogene function of heterogeneous nuclear ribonucleoprotein A1 overexpression in HBV-related hepatocellular carcinoma. Int J Biol Macromol. 2019; 129: 140-51.

47. Zhou HC, Fang JH, Shang LR, Zhang ZJ, Sang Y, Xu L, et al, MicroRNAs miR-125b and miR-100 suppress metastasis of hepatocellular carcinoma by disrupting the formation of vessels that encapsulate tumour clusters. J Pathol. 2016; 240: 450-60.

48. Ge YY, Shi Q, Zheng ZY, Gong J, Zeng C, Yang J, et al. MicroRNA-100 promotes the autophagy of hepatocellular carcinoma cells by inhibiting the expression of mTOR and IGF-1R. Oncotarget. 2014; 5: 6218-28.

49. Ye Y, Li SL, Wang JJ. miR-100-5p Downregulates mTOR to Suppress the Proliferation, Migration, and Invasion of Prostate Cancer Cells. Front Oncol. 2020; 10: 578948

50. Yu Z, Li N, Jiang K, Zhang N, Yao LL. MiR-100 up-regulation enhanced cell autophagy and apoptosis induced by cisplatin in osteosarcoma by targeting mTOR. Eur Rev Med Pharmacol Sci. 2020; 24: 7570.

51. Lin L, Huang Y, Zhuang W, Lin P, Ma X. miR-100 inhibits cell proliferation in mantle cell lymphoma by targeting mTOR. Exp Hematol Oncol. 2020; 9: 25. 
52. Ge YY, Shi Q, Zheng ZY, Gong J, Zeng C, Yang J, et al. MicroRNA-100 promotes the autophagy of hepatocellular carcinoma cells by inhibiting the expression of mTOR and IGF-1R. Oncotarget. 2014; 5: 6218-28. 